\title{
Efficient Electrocatalytic Upgrading of Furan-Based Biomass: Key Roles of Two-Dimensional Mesoporous Heterostructure and Ternary Electrolyte
}

\author{
Haoran Wu \\ Shanghai Jiao Tong University \\ Yashi Zou \\ Shanghai Jiao Tong University \\ Haishan Xu \\ Shanghai Jiao Tong University \\ Jianming Yang
}

School of Physics and Electronic Science, East China Normal University

Qinye Bao

East China Normal University

Liang Wu

Shanghai Jiao Tong University https://orcid.org/0000-0001-8737-0562

Yiyong Mai ( $\nabla$ mai@sjtu.edu.cn )

Shanghai Jiao Tong University https://orcid.org/0000-0002-6373-2597

\section{Article}

Keywords: electrocatalytic systems, catalytic performance, biomass

Posted Date: April 23rd, 2021

DOI: https://doi.org/10.21203/rs.3.rs-424248/v1

License: (c) (i) This work is licensed under a Creative Commons Attribution 4.0 International License. Read Full License 


\title{
Efficient Electrocatalytic Upgrading of Furan-Based Biomass: Key Roles of Two-Dimensional Mesoporous Heterostructure and Ternary Electrolyte
}

Haoran Wu, ${ }^{1}$ Yashi Zou, ${ }^{1}$ Haishan Xu, ${ }^{1}$ Jianming Yang, ${ }^{2}$ Qinye Bao, ${ }^{2}$ Liang Wu, ${ }^{1}$ and Yiyong Mai ${ }^{1} *$ ${ }^{1}$ School of Chemistry and Chemical Engineering, Frontiers Science Center for Transformative Molecules, Shanghai Key Laboratory of Electrical Insulation and Thermal Ageing, Shanghai Jiao Tong University, 800 Dongchuan Road, Shanghai 200240, P. R. China.

*E-mail: mai@sjtu.edu.cn

${ }^{2}$ Key Laboratory of Polar Materials and Devices, School of Physics and Electronic Science, East China Normal University, Shanghai 200241, P. R. China.

\begin{abstract}
Development of high-performance electrocatalytic systems for efficient conversion of biomass to value-added chemicals under mild conditions and understanding of their mechanisms are of profound significance, but have remained a great challenge. Here, we report the first development of two-dimensional mesoporous electrocatalyst for biomass conversion. The electrocatalyst (meso-PA/PmPD/GO) consists of phytic acid (PA)-doped mesoporous poly(m-phenylenediamine) layers coated on graphene oxide nanosheets. Meanwhile, a high-performance ternary electrolyte containing 1-butyl-3-methylimidazolium tetrafluoroborate $\left(\mathrm{BmimBF}_{4}\right)$, acetonitrile and $\mathrm{H}_{2} \mathrm{O}$ is developed. The combination of meso-PA/PmPD/GO and the ternary electrolyte realizes highly efficient conversion of two important biomass derivatives at room temperature. One involves a hardly achieved oxidation of furfuryl alcohol to 6-hydroxy-2,3-dihydro-6H-pyrano-3-one with high faradic efficiency (FE: 83.7\%) and selectivity $(87.9 \%)$. The other involves the oxidation of furfural to 5-hydroxy-2(5H)-furanone with record-high FE (98.9\%) and selectivity (93.6\%). Mechanism study including DFT calculations unveils that $\mathrm{N}$-heterocyclic carbenes $\left(\mathrm{Bmim}^{*}\right)$ generated from $\mathrm{BmimBF}_{4}$ act as the reaction-determining active species. Additionally, the synergistic effect of the PA doping, mesoporous structure and p-n heterojunction interface in meso-PA/PmPD/GO favors the mass transport and the transfer of generated holes to the outer layers, thus boosting the catalytic performance.
\end{abstract}


With the ever-increasing depletion of global fossil resources, the utilization of green and renewable biomass to produce high value-added chemicals has attracted tremendous interest. ${ }^{1-5}$ Among different types of biomass (e.g., lignocellulose, triglycerides, chitin, starch), lignocellulose is the most abundant resource. Derived from lignocellulose, furfuryl alcohol and furfural compounds have been recognized as indispensable and renewable feedstocks for sustainable chemical productions. ${ }^{6}$ Compared with traditional thermocatalytic reactions, electrocatalytic oxidation represents a promising strategy for biomass conversion due to its mild reaction conditions including under ambient conditions and using $\mathrm{H}_{2} \mathrm{O}$ as the green oxygen source, etc. ${ }^{7-12}$ However, at present most of electrocatalytic biomass conversions rely on metal-based catalysts; ${ }^{13-15}$ numerous biomass conversions to high value-added chemicals have been preliminarily realized with low faradaic efficiency (FE)/selectivity, or even have been hardly achieved. For example, 6-hydroxy-2,3-dihydro-6H-pyrano-3-one (HDPO) is a key component in many biologically active compounds or the intermediates of numerous pharmaceuticals. ${ }^{16-18}$ It has been primarily prepared from furan derivatives via thermal catalysis, which involves harsh conditions $\left(-70{ }^{\circ} \mathrm{C}\right)$ and/or toxic oxidants. $^{19,20}$ Only one successful case was reported very recently on the electrocatalytic conversion of biomass (furfuryl alcohol) to HDPO by employing Ni-based catalyst. ${ }^{21}$ It remains challenging to develop new electrocatalytic systems for achieving high FE/selectivity and understand the complicated reaction mechanism. On the other hand, for another important electrocatalytic oxidation of furfural to 5-hydroxy-2(5H)-furanone (HFO), which is a critical intermediate for the synthesis of pharmaceuticals, rubber additives, and agrochemical compounds, ${ }^{22}$ it has still remained unsolved to simultaneously achieve both high FE and selectivity for HFO; the highest FEs of all the reported routes have reached only $\sim 60 \%$ with selectivity of $<83 \%$. $^{23-26}$

The aforementioned problems mainly originate from the low utilization ratio of catalysts, 
because for most of reported electrocatalysts only the catalytic sites on the surface can be used. Recently, two-dimensional (2D) mesoporous materials have aroused great attention in the field of electrocatalysis, in light of their high specific surface areas (SSAs), abundant catalytic sites, and short charge transport distance. ${ }^{27-29}$ The mesopores enable the mass transportation into the internal of electrocatalysts and thus increase the utilization ratio of active sites, leading to high catalytic performance. However, to our knowledge, 2D mesoporous materials have not yet been explored as electrocatalysts for biomass conversion. This vacancy inspires the interest of study.

On the other hand, the efficiency of an electrocatalytic process also depends strongly on the electrolyte system. ${ }^{30}$ Traditional electrolytes for biomass conversion are usually based on aqueous inorganic salt (such as $\mathrm{K}^{+}, \mathrm{Na}^{+}$, and $\mathrm{NH}_{4}{ }^{+}$, etc.) systems. However, these systems usually undergo severely competitive hydrogen or oxygen evolution effects. Furthermore, biomass derivatives usually possess multi-functional groups, which are difficult to be selectively activated in aqueous inorganic salt systems. Therefore, the development of high-performance electrolyte systems is also an essential branch in the area of biomass conversion.

Here, we develop a 2D metal-free heterostructural electrocatalyst (denoted as meso-PA/PmPD/GO), which consists of phytic acid (PA)-doped mesoporous poly(m-phenylenediamine) (PmPD) conductive layers coated on both surfaces of graphene oxide (GO) nanosheets, through a block copolymer templated interfacial self-assembly strategy (Fig. 1). The meso-PA/PmPD/GO nanosheets possess in-plane cylindrical mesopores with an average diameter of $10 \pm 2 \mathrm{~nm}$, a thickness of ca. $30 \mathrm{~nm}$, and a high specific surface area of $258 \mathrm{~m}^{2} \mathrm{~g}^{-1}$. Meanwhile, we also develop an efficient ternary electrolyte system containing 1-butyl-3-methylimidazolium tetrafluoroborate $\left(\mathrm{BmimBF}_{4}\right)$, acetonitrile and $\mathrm{H}_{2} \mathrm{O}$. Strikingly, the combination of the meso-PA/PmPD/GO nanosheets and the ternary electrolyte enables excellent 


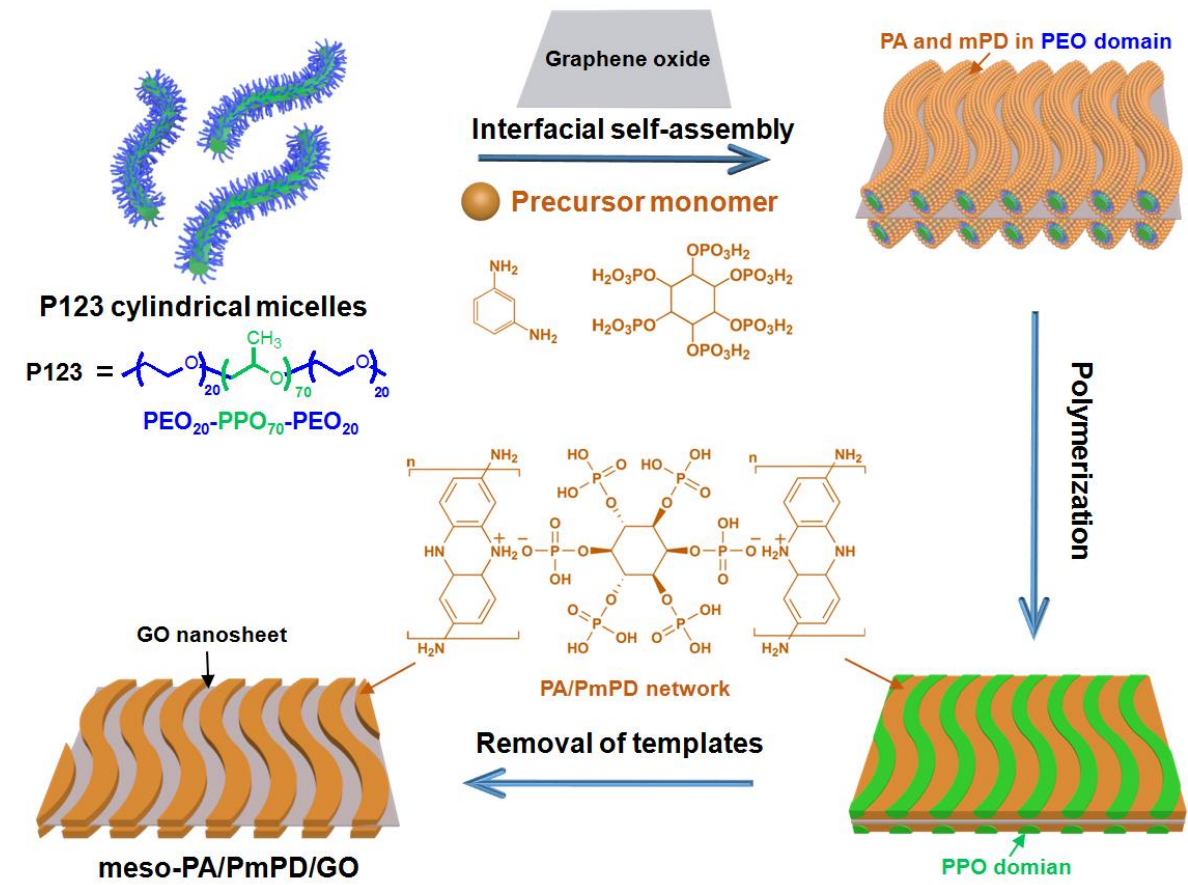

Fig. 1 Schematic illustration of the synthesis of meso-PA/PmPD/GO nanosheets with in-plane cylindrical mesopores through an interfacial self-assembly strategy.

a

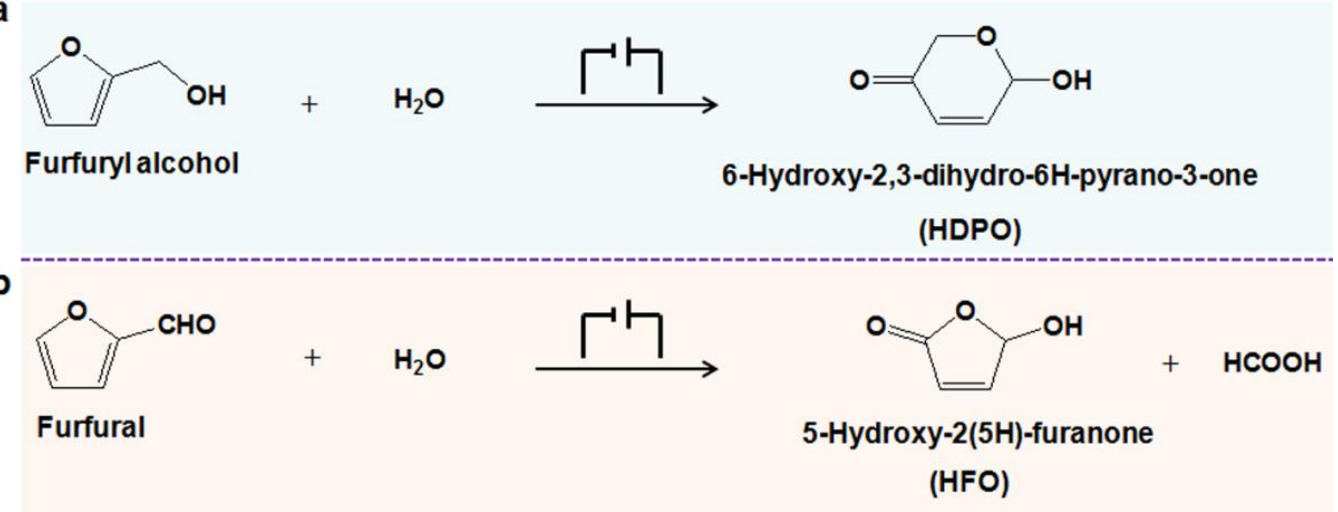

Scheme 1. Electrochemical oxidations of furfuryl alcohol to HDPO (a) and furfural to HFO (b).

electrocatalytic activity for the biomass conversions of both furfuryl alcohol to HDPO and furfural to HFO using water as the green oxygen source at room temperature (Scheme 1). For the hardly achieved oxidation of furfuryl alcohol to HDPO, the new electrocatalytic system realizes very high FE of $83.7 \%$ and selectivity of $87.9 \%$. For the oxidation of furfural to HFO, record-high FE of $98.9 \%$ and selectivity of $93.6 \%$ is achieved, which are much higher than all of the reported values. Mechanism study including density functional theory (DFT) calculations unveil that N-heterocyclic carbenes $(\mathrm{Bmim} *)$ originated from $\mathrm{BmimBF}_{4}$ act as the reaction-determining active species. 
Meanwhile, the synergetic effect of the doped PA, mesoporous structure, and p-n heterojunction interface in the meso-PA/PmPD/GO nanosheets favors the mass transport, the electron-hole separation, and the transfer of holes to the outer layers, thus optimizing the catalytic performance. This study opens a new avenue for the development of high-performance electrocatalytic systems for biomass conversion, and provides insightful clues for understanding complicated mechanisms of biomass conversion.

\section{Results and Discussion}

Preparation and characterizations of meso-PA/PmPD/GO nanosheets. The meso-PA/PmPD/GO nanosheets were prepared through a block copolymer templated interfacial self-assembly strategy ${ }^{31-37}$, which employed Pluronic P123 copolymer (poly(ethylene oxide $)_{20}$-block-poly(phenylene oxide $)_{70}$-block-poly(ethylene oxide $)_{20}$, the subscript numbers denote the degrees of polymerization) as the pore-creating template, m-phenylenediamine (mPD) as the monomer, PA as the dopant, and GO of ca. $1 \mathrm{~nm}$ thickness as the 2D substrate, respectively (Fig. 1). Typically, the P123 copolymer was firstly dissolved in water following reported procedures, forming cylindrical micelles. ${ }^{38}$ After mixing the aqueous solution of the micelles and that of free-standing GO nanosheets, mPD and PA, the hydrogen-bonding and electrostatic interactions among these precursors could drive the close packing of the cylindrical micelles on the surfaces of GO along with the adsorption of mPD and PA in the poly(ethylene oxide) (PEO) domains. ${ }^{39-41}$ The subsequent polymerization of mPD coupled with the PA doping was confined in the PEO regions, leading to the formation of PA-doped PmPD (PA/PmPD) networks on the GO surfaces. After the removal of the copolymer template, 2D heterostructural nanosheets composed of two mesoporous polymeric PA/PmPD layers with cylindrical pores sandwiching the GO nanosheet are obtained. 
Scanning electron microscopy (SEM) and transmission electron microscopy (TEM) images of the meso-PA/PmPD/GO nanocomposite clearly show a flat sheet-like structure with closely-packed in-plane cylindrical mesopores (Fig. 2a,b and Supplementary Fig. S1). The average pore diameter measured from either SEM or TEM image is $10 \pm 2 \mathrm{~nm}$. Atomic force microscopy (AFM) height profile gives an average thickness of ca. $30 \mathrm{~nm}$ for the meso-PA/PmPD/GO nanosheets (Fig. 2c). $\mathrm{N}_{2}$ adsorption-desorption analysis (Supplementary Fig. S2) reveals that the meso-PA/PmPD/GO nanosheets possess a high specific surface area of $258 \mathrm{~m}^{2} \mathrm{~g}^{-1}$ (based on the Brunauer-Emmett-Teller method) and a mean mesopore size of ca. $12 \mathrm{~nm}$ (using the Barrett-Joyner-Halenda approach). Fourier transform infrared (FTIR) and Raman spectra of meso-PA/PmPD/GO show the characteristic signals originated from the phosphate group, imine/amine group, and GO (Supplementary Figs. S3 and S4), suggesting the incorporation of PA in the nanosheets. The elemental mapping images show a uniform distribution of $\mathrm{C}, \mathrm{N}, \mathrm{O}$, and $\mathrm{P}$ elements in the meso-PA/PmPD/GO sheets (Figs. 2d-2h). Inductively coupled plasma (ICP) spectroscopy analysis gives a PA content of $13.1 \mathrm{wt} \%$ for meso-PA/PmPD/GO based on the detected P content of $3.7 \mathrm{wt} \%$. X-ray diffraction (XRD) pattern shows weak and broad diffraction peaks (Supplementary Fig. S5),
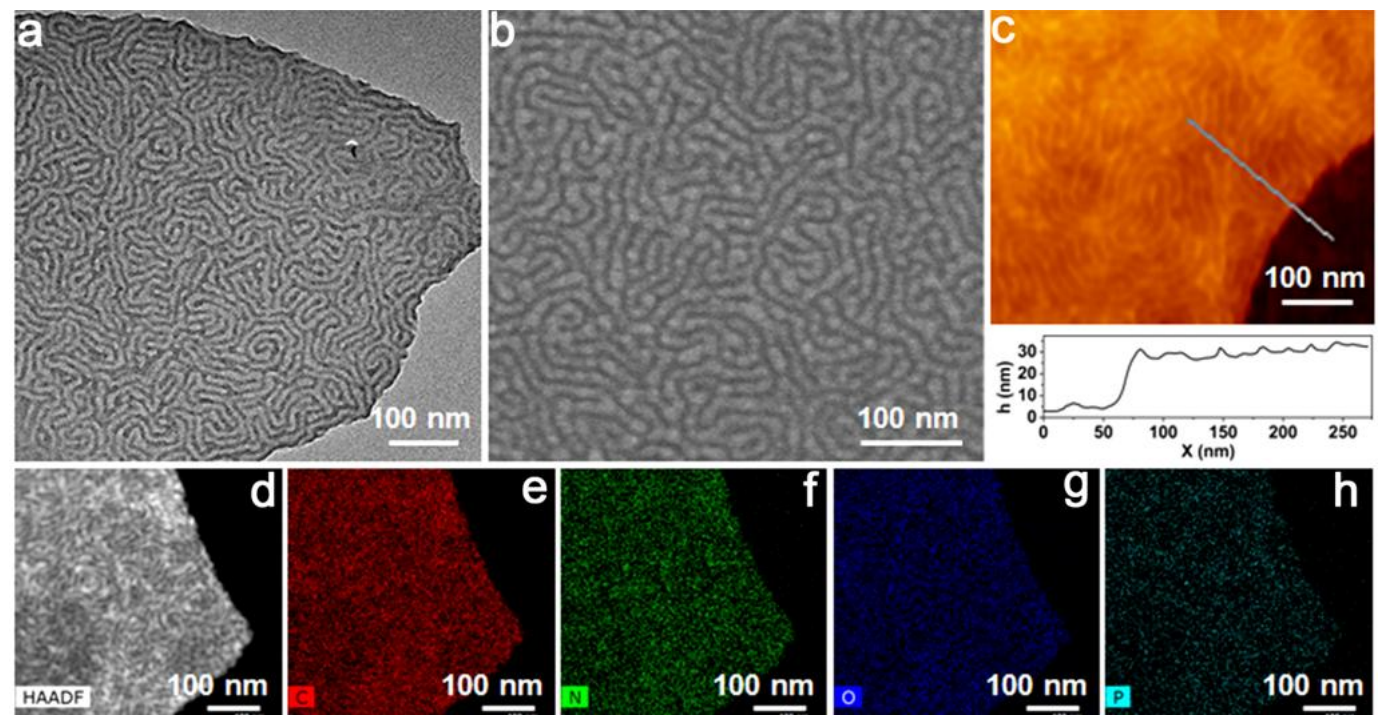

Fig. 2 Structural characterizations of the meso-PA/PmPD/GO nanosheets. (a) TEM image; (b) SEM image; (c) AFM image; (d-h) element mapping images. 
indicating an amorphous feature of meso-PA/PmPD/GO.

\section{Electrocatalytic performance of meso-PA/PmPD/GO for the conversion of furan-based}

biomass. The electrocatalytic performance of meso-PA/PmPD/GO was first evaluated for the oxidation of furfuryl alcohol. A ternary electrolyte containing $\mathrm{BmimBF}_{4}$, acetonitrile and $\mathrm{H}_{2} \mathrm{O}$ was employed, in view of the wide electrochemical window, good electrical conductivity and negligible vapour pressure of $\mathrm{BmimBF}_{4} \cdot{ }^{42}$ Linear sweep voltammetry (LSV) curve shows a notable increase in the current density at the potential of $>1.8 \mathrm{~V}$ vs NHE when furfuryl alcohol was present in the ternary electrolyte (Fig. 3a), suggesting the occurrence of furfuryl alcohol oxidation. ${ }^{1} \mathrm{H}$ NMR analysis revealed the generation of HDPO as the major product (Scheme 1a), along with the coexistence of a small amount of furfural and formic acid $(\mathrm{HCOOH})$ as the byproducts (Supplementary Fig. S6).

Since the electrolyte composition plays a key role in electrocatalytic conversion of biomass, the effect of anolyte composition on the furfuryl alcohol oxidation was evaluated under the optimal potential of 2.3 V vs NHE (see Supplementary Fig. S7 and the discussion in Page S6 for exploring the optimal potential). The results show that a low concentration $(0.85 \mathrm{wt} \%)$ of $\mathrm{BmimBF}_{4}$ is not favorable for the conversion of furfuryl alcohol (Fig. 3b), probably because of the poor conductivity of the electrolyte without a sufficient amount of $\mathrm{BmimBF}_{4}$. However, too high concentrations (> 3.4 $\mathrm{wt} \%$ ) of $\mathrm{BmimBF}_{4}$ induce the generation of furfural, which can be further oxidized to produce $\mathrm{HCOOH}$. Through an overall tradeoff, $3.4 \mathrm{wt} \%$ is selected as the optimal concentration of $\mathrm{BmimBF}_{4}$ in the anolyte. On the other hand, $\mathrm{H}_{2} \mathrm{O}$ as green oxygen source is indispensable for the oxidation of furfuryl alcohol. In our system, a very low conversion (37.4 wt\%) of furfuryl acohol is observed at a low $\mathrm{H}_{2} \mathrm{O}$ concentration $(0.85 \mathrm{wt} \%$, Fig. $3 \mathrm{c})$. The conversion rate increases with increasing the $\mathrm{H}_{2} \mathrm{O}$ concentration in the range of 0.85-5.1 wt\%, whereas the selectivity and FE of 
furfural decrease gradually due to the further oxidation of furfural. With a further increase in the $\mathrm{H}_{2} \mathrm{O}$ concentration, no obvious changes are observed in the FE and selectivity of HDPO. Therefore, $5.1 \mathrm{wt} \%$ is the optimal $\mathrm{H}_{2} \mathrm{O}$ concentration and selected for the subsequent testing. As comparison, traditional aqueous inorganic salt electrolytes, such as $\mathrm{KH}_{2} \mathrm{PO}_{4}, \mathrm{NaNO}_{3}$, and $\left(\mathrm{NH}_{4}\right)_{2} \mathrm{SO}_{4}$ systems, were also evaluated for the electrochemical oxidation of furfuryl alcohol (Supplementary Table S1). However, very low FE and selectivity of HDPO were obtained in these electrolyte systems, reflecting a crucial role of the ternary electrolyte in the highly efficient electrooxidation of furfuryl alcohol.
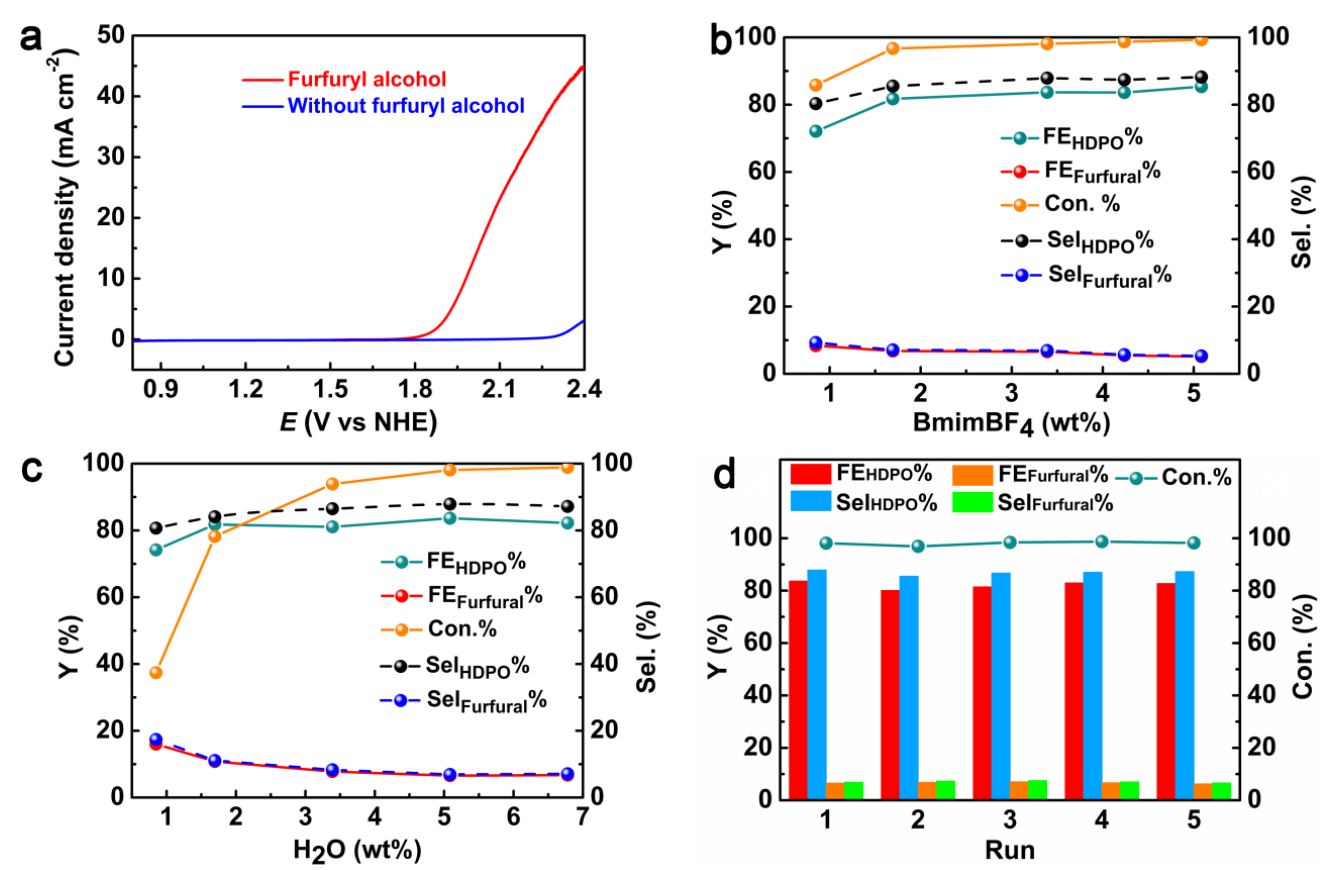

Fig. 3 Effect of various parameters on the electrochemical oxidation of furfuryl acohol to HDPO over the meso-PmPD/PA/GO catalyst. (a) LSV measurement; (b) $\mathrm{BmimBF}_{4}$ content; (c) $\mathrm{H}_{2} \mathrm{O}$ content; (d) cycling performance.

Additionally, the electrocatalytic performance of two control samples, including nonporous $\mathrm{PA} / \mathrm{PmPD} / \mathrm{GO}$ (abbreviated as non-PA/PmPD/GO) and mesoporous PmPD/GO (meso-PmPD/GO) nanosheets without the PA doping, was compared for the oxidation of furfuryl alcohol under the optimal conditions (Table 1). The structural characterizations of the control samples are given in the Supplementary Figs. S8 and S9. It is noted that among all of the samples, meso-PA/PmPD/GO 
exhibits the best performance with strikingly high FE (83.7\%) and selectivity $(87.9 \%)$ of HDPO (Table 1). In addition, the performance differences suggest that both the PA incorporation and the mesoporous structure contribute remarkably to the increase of FE and selectivity.

Table 1. Electrochemical oxidations of furfuryl alcohol over different electrocatalysts at $2.3 \mathrm{~V}$ vs NHE in the ternary electrolyte of $\mathrm{BmimBF}_{4}(3.4 \mathrm{wt} \%)-\mathrm{MeCN}_{-} \mathrm{H}_{2} \mathrm{O}(5.1 \mathrm{wt} \%)$.

\begin{tabular}{lccccccc}
\hline Samples & $\mathrm{d}(\mathrm{nm})$ & $\mathrm{SSA}\left(\mathrm{m}^{2} \mathrm{~g}^{-1}\right)$ & $\mathrm{Con} .(\%)$ & $\mathrm{HDPO}_{\mathrm{FE} \%}$ & $\mathrm{HDPO}_{\mathrm{Sel} . \%}$ & furfural \\
\hline $\mathrm{FE} \%$ & furfural $_{\text {Sel. } \%}$ \\
\hline meso-PA/PmPD/GO & $10 \pm 2$ & 258 & 98.1 & 83.7 & 87.9 & 6.6 & 6.9 \\
\hline moso-PmPD/GO & $11 \pm 1$ & 224 & 84.5 & 66.4 & 76.0 & 13.3 & 15.2 \\
\hline
\end{tabular}

$\mathrm{d}$ denotes the average pore diameter obtained from TEM images; SSA represents the specific surface area obtained from the nitrogen adsorption-desorption isotherms by the BET method; Con. is the abbreviation of conversion.

Next, the cycling stability of meso-PA/PmPD/GO was examined. Fig. 3d shows no obvious decrease in the furfuryl alcohol conversion, FE and selectivity of HDPO after five catalytic cycles. SEM and FTIR analyses reveal no obvious differences in the morphology and main characteristic vibration peaks of meso-PA/PmPD/GO after the catalytic cycles (Supplementary Figs. S10 and S11), suggesting the excellent cycling stability of meso-PA/PmPD/GO.

During the electrochemical oxidation of furfuryl alcohol, we also found that the furfural product could be further oxidized at higher potentials $(>2.3 \mathrm{~V}$ vs NHE) in the ternary electrolyte. Therefore, the electrochemical oxidation of furfural was also studied in the present work (Fig. 4a). ${ }^{1} \mathrm{H}$ NMR analysis revealed that $\mathrm{HFO}$ and $\mathrm{HCOOH}$ were the two main products of this conversion (Scheme 1b), along with a very small amount of maleic acid as byproduct (Supplementary Fig. S12). Impressively, the selectivity of $\mathrm{HCOOH}$ reached over 97\%, while record-high $\mathrm{FE}(98.9 \%)$ and selectivity (93.6\%) of HFO were also achieved at the optimal potential of $2.45 \mathrm{~V}$ vs NHE in our system (Fig. 4a). The cycling performance testing proved the excellent catalytic stability of 
meso-PA/PmPD/GO in the oxidation of furfural to HFO (Fig. 4b).

Similar to the electrocatalytic oxidation of furfuryl alcohol, the ternary electrolyte system also enables evidently higher FE and selectivity in the oxidation of furfural to HFO in comparison to those obtained in the aqueous inorganic salt electrolyte systems (Supplementary Table S2). In addition, among all the evaluated samples, meso-PA/PmPD/GO exhibits the best catalytic performance (Table 2). As well, the performance differences indicate the notable contributions of the PA doping and the mesoporous structure to the high electrocatalytic performance of meso-PA/PmPD/GO.
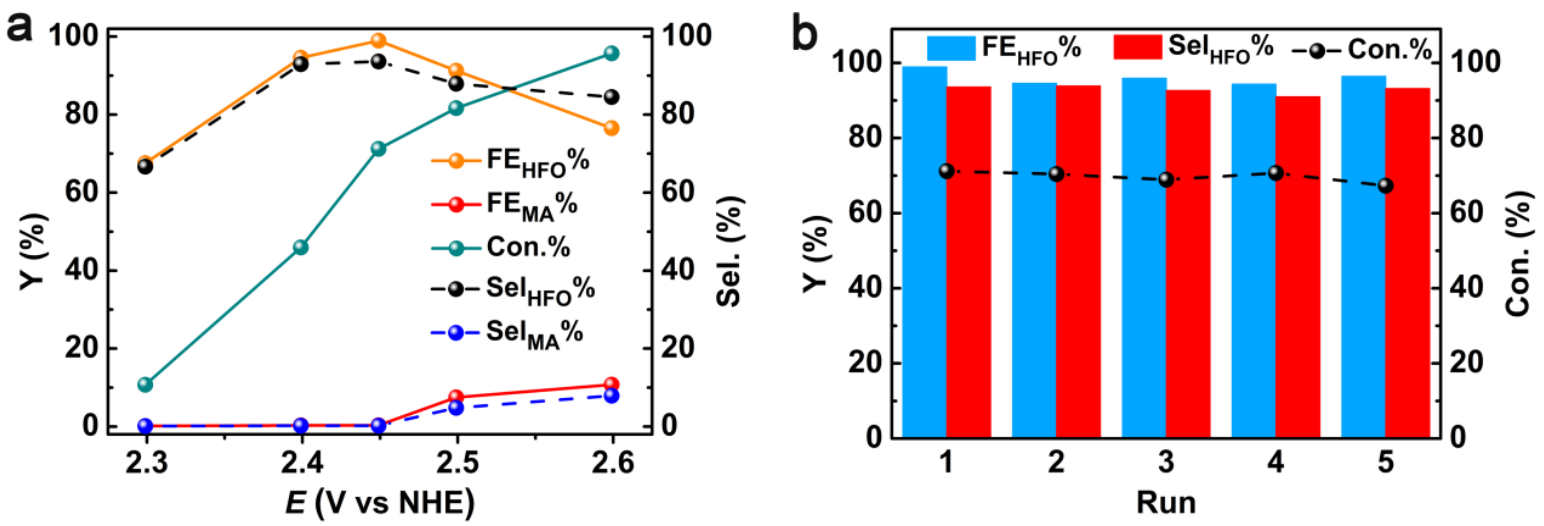

Fig. 4 The electrochemical oxidation of furfural over the meso-PA/PmPD/GO catalyst in the ternary electrolyte. (a) Applied potential and (b) cycling performance.

Table 2. Electrochemical oxidations of furfural to HFO over different electrocatalysts at $2.45 \mathrm{~V}$ vs NHE in the ternary electrolyte.

\begin{tabular}{lccc}
\hline Samples & Con. $(\%)$ & $\mathrm{HFO}_{\mathrm{FE} \%}$ & $\mathrm{HFO}_{\text {Sel. } \%}$ \\
\hline meso-PA/PmPD/GO & 71.2 & 98.9 & 93.6 \\
\hline meso-PmPD/GO & 41.6 & 72.5 & 76.8 \\
\hline non-PA/PmPD/GO & 56.3 & 83.8 & 84.1 \\
\hline
\end{tabular}

Mechanism investigation. To gain an insight into the reaction mechanisms of the electrocatalytic oxidations of furfuryl alcohol and furfural, the reaction pathways were investigated. For the electrochemical oxidation of furfuryl alcohol, a reasonable mechanism involving two pathways (Pathway I and II) is proposed in Fig. 5a, based on the fact that the major product HDPO along with 
byproducts including furfural and $\mathrm{HCOOH}$ was detected. In pathway I (as the main reaction), the furfuryl alcohol undergoes the opening and isomerization of furan ring to generate an intermediate $\mathbf{1}$. Due to the existence of $-\mathrm{OH}$ and $-\mathrm{CHO}$ in 1, an intramolecular aldol condensation can take place quickly to form an intermediate 2. Then, HDPO is formed by the oxidation of 2 . As the side reaction, the pathway II produces a small amount of furfural. Density functional theory (DFT) calculations were conducted using the Gaussian 09 program $^{43}$ to verify the reaction mechanism. The transient state (TS) between corresponding reactant and product at each reaction step were confirmed by IRC (intrinsic reaction coordinate) calculations. All structures were optimized at B3LYP/6-311G(d,p) level. The results are summarized in Fig. 5b. The calculated energy profiles of the reaction pathways with detailed reactants, products, and intermediates are presented in the Supplementary Information (Supplementary Figs. S13-S15 and Table S3). In Fig. 5b and Supplementary Fig. S16, two series of intermediate states (IM) with different configurations (diastereoisomer) are involved as pathway I(a) and I(b), respectively. The pathway I consists of two stages. In stage A (furfuryl alcohol to IM1-3), the furfuryl alcohol molecule is subjected to the attack of $\mathrm{H}_{2} \mathrm{O}$ molecule to generate IM1-1. Subsequently, the isomerization of IM1-1 yields IM1-2 and then its intramolecular condensation generates IM1-3. In stage B (IM1-3 to HDPO), the Bmim* active species generated from $\mathrm{Bmim}^{+}\left(\mathrm{i} . \mathrm{e} . \mathrm{Bmim}^{+}+\mathrm{BF}_{4}^{-}=\mathrm{Bmim}^{*}+\mathrm{HBF}_{4}\right.$ ), which is a weak equilibrium, ${ }^{44-46}$ initiate the oxidation of IM1-3, passing through TS1-1, to generate HDPO and BmimH. Afterwards, BmimH is oxidized back to $\mathrm{Bmim}^{+}$on the electrode surface to complete the $\mathrm{Bmim}^{+}$-to-Bmim*-to-BmimH-to-Bmim ${ }^{+}$cycle. In addition, the DFT results indicate that the furfuryl alcohol reacts with Bmim* to form furfural (Supplementary Fig. S17), supporting the coexistence of pathway II. However, the barrier energy for the transition state in pathway II (TS2-1, $24.3 \mathrm{kcal} / \mathrm{mol})$ is higher than that of TS1-1a $(23.8 \mathrm{kcal} / \mathrm{mol})$, accounting for pathway II as the side 
reaction route as we found in experiment. Meanwhile, there is no side reaction of Bmim* in the ternary electrolyte, which contributes to the high FE and selectivity of HDPO. Nevertheless, in the aqueous inorganic salt electrolyte systems, the large amount of hydroxyl radicals generated from the electrolysis of water can induce a side reaction of oxygen evolution, ${ }^{47,48}$ leading to much lower FE and selectivity.
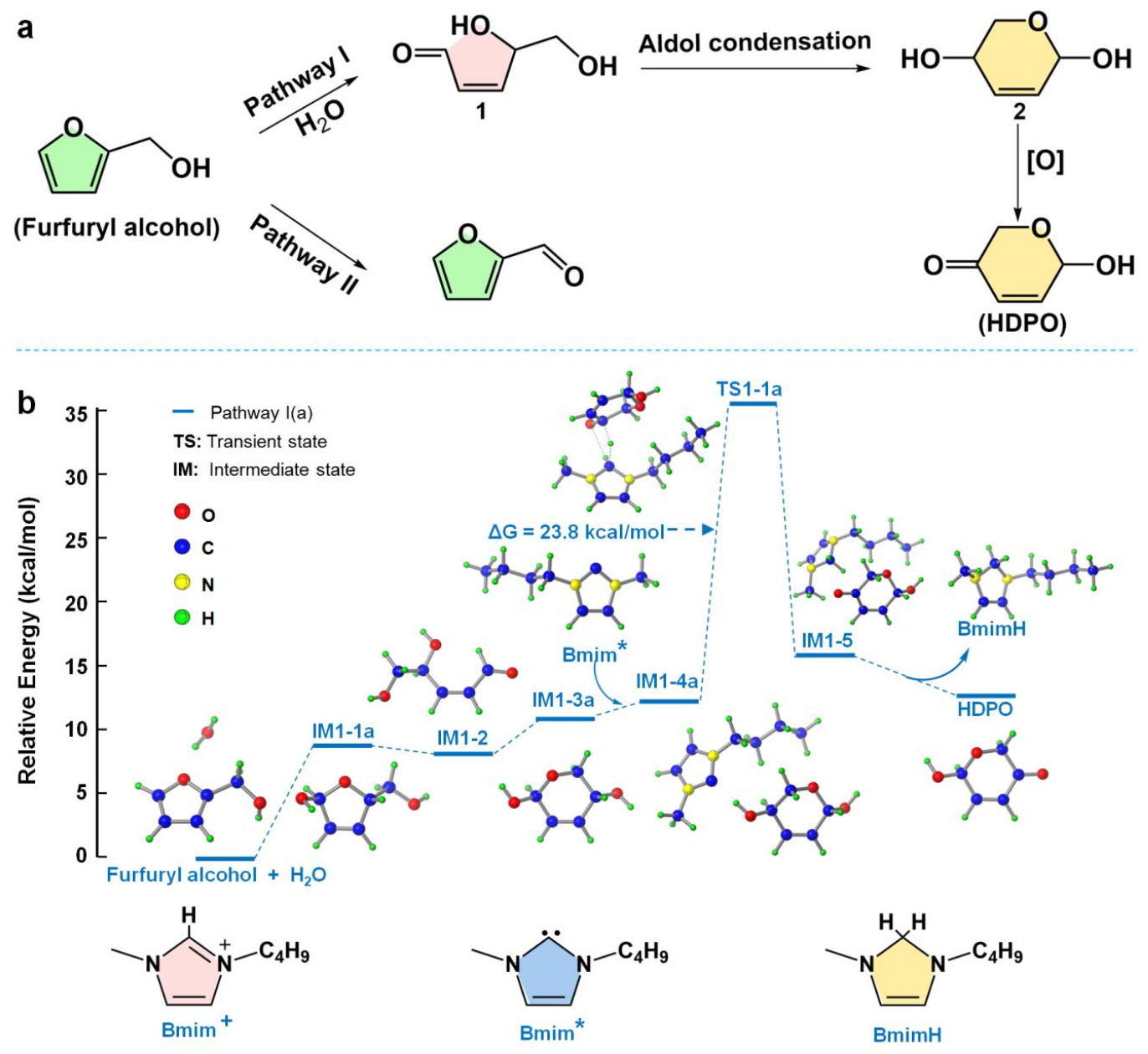

Fig. 5 (a) Reaction pathways for the electrochemical oxidation of furfuryl alcohol over the meso-PA/PmPD/GO electrocatalyst in the ternary electrolyte. (b) The corresponding DFT calculations.

The reaction mechanism of the electrochemical oxidation of furfural to HFO was firstly explored by employing 5-methylfurfural (highly similar to furfural) as the reactant. The results and the corresponding reaction pathway are presented in the Supplementary Information (see Supplementary Figs. S18, S19 and the discussion in Page S14). Accordingly, the reaction pathway 
for the oxidation of furfural to HFO is proposed in Fig. 6a. Initially, an intermediate $\mathbf{3}$ and HCOOH are generated via the cleavage of the aldehyde group in furfural, and the unstable $\mathbf{3}$ quickly rearranges to an intermediate 4 . Afterwards, 4 undergoes the ring opening to form an intermediate 5, which is converted to an intermediate 6 through hydroxyl oxidation (Supplementary Fig. S20). Finally, HFO is produced through the isomerization of $6 .^{49}$ DFT calculations (Fig. 6b) show that four reaction stages are involved. Stage A (furfural to IM3-3): the furfural is subjected to the attack of hydroxyl radical to form $\mathrm{HCOOH}$ and IM3-3. Stage B (IM3-3 to IM3-4): IM3-4 is formed via the hydrolysis of IM3-3. Stage C (IM3-4 to IM3-7): Bmim* initiates the oxidation of IM3-4, passing through TS3-2, to generate IM3-7; this key stage determines the high FE and selectivity of HFO. Finally (Stage D), HFO is formed via the isomerization of IM3-7.

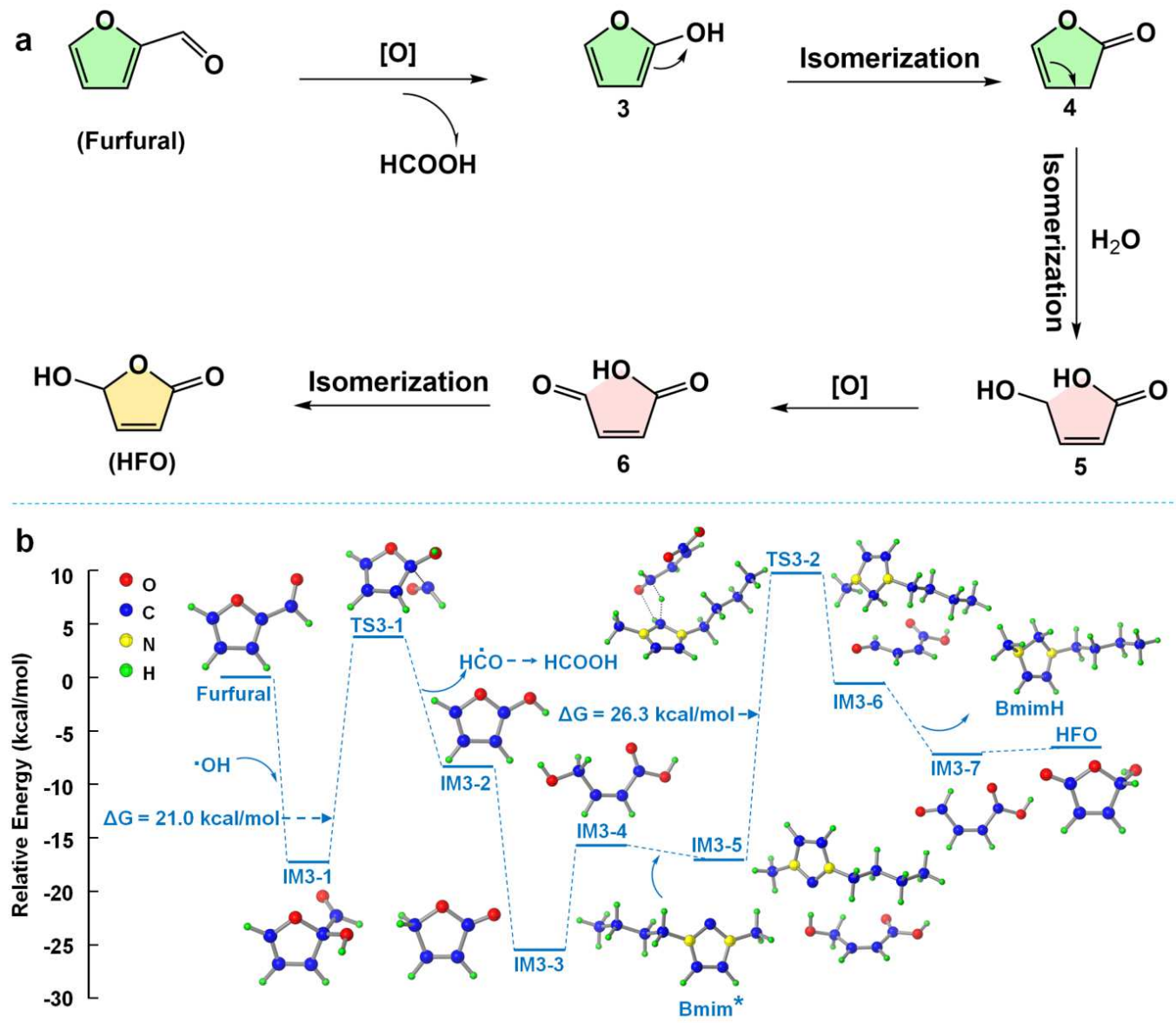

Fig. 6 (a) Reaction pathways for the electrochemical oxidation of furfural over the meso-PA/PmPD/GO electrocatalyst in the ternary electrolyte. (b) The corresponding DFT calculations. 
The aforementioned results indicate that $\mathrm{Bmim}^{*}$ originated from $\mathrm{BmimBF}_{4}$ is crucial in the electrochemical oxidation of furfuryl alcohol and furfural. We conducted ${ }^{1} \mathrm{H}$ NMR measurement to validate the vital role of $\mathrm{BmimBF}_{4}$. The ${ }^{1} \mathrm{H}$ NMR spectra of the mixture of $\mathrm{BmimBF}_{4}$ and furfuryl alcohol in DMSO- $d_{6}$ show that the chemical shifts of the furan ring $(\mathrm{H}-1, \mathrm{H}-2, \mathrm{H}-5)$ in furfuryl alcohol move to the high field with an increase in the $\mathrm{BmimBF}_{4}$ concentration; meanwhile the chemical shifts of the aromatic imidazolium ring $(\mathrm{H}-\mathrm{a}, \mathrm{H}-\mathrm{b}, \mathrm{H}-\mathrm{c})$ in $\mathrm{BmimBF}_{4}$ also move apparently to the high field (Supplementary Fig. S21). This result indicates the presence of $\pi-\pi$ interaction between the imidazolium ring and the furan ring, ${ }^{50}$ which contributes to boosting the oxidation of furfuryl alcohol. A similar phenomenon is also observed in the ${ }^{1} \mathrm{H}$ NMR spectrum of the mixture of $\mathrm{BmimBF}_{4}$ and furfural (Supplementary Fig. S22), in which the chemical shift of aldehyde group in furfural shows a high field shift. The reason can be attributed to the $\pi-\pi$ interaction between the imidazolium ring and the furan ring of furfural. ${ }^{50}$ Furthermore, Tafel plots were employed to probe the reaction kinetics. ${ }^{51,52}$ The Tafel slopes obtained in the ternary electrolyte are smaller than those measured in the aqueous inorganic salts systems (Fig. 7a,b). This result proves the faster reaction kinetics in the ternary electrolyte system, which is favorable for the enhancement of the electrocatalytic performance.

Generally, the structure of electrocatalyst affects its catalytic performance. In our case, meso-PA/mPDA/GO shows better electrocatalytic performance than that of non-PA/mPDA/GO, which profits from its mesoporous structure with in-plane cylindrical pores. The in-plane cylindrical mesopores can afford meso-PA/mPDA/GO a higher specific surface area, and also facilitate the mass transport along the cylindrical pores into the stacked 2D nanosheets (see Fig. 8 for illustration). As a line of evidence, the lower slope of the $Z^{\prime}-\omega^{-1 / 2}$ line of meso-PA/PmPD/GO at the low-frequency region demonstrates that meso-PA/PmPD/GO is more favorable for mass transport, 
in this case the ternary electrolyte and the biomass molecules, in comparison to non-PA/PmPD/GO under similar conditions (Fig. 7c,d and Supplementary Fig. S23).
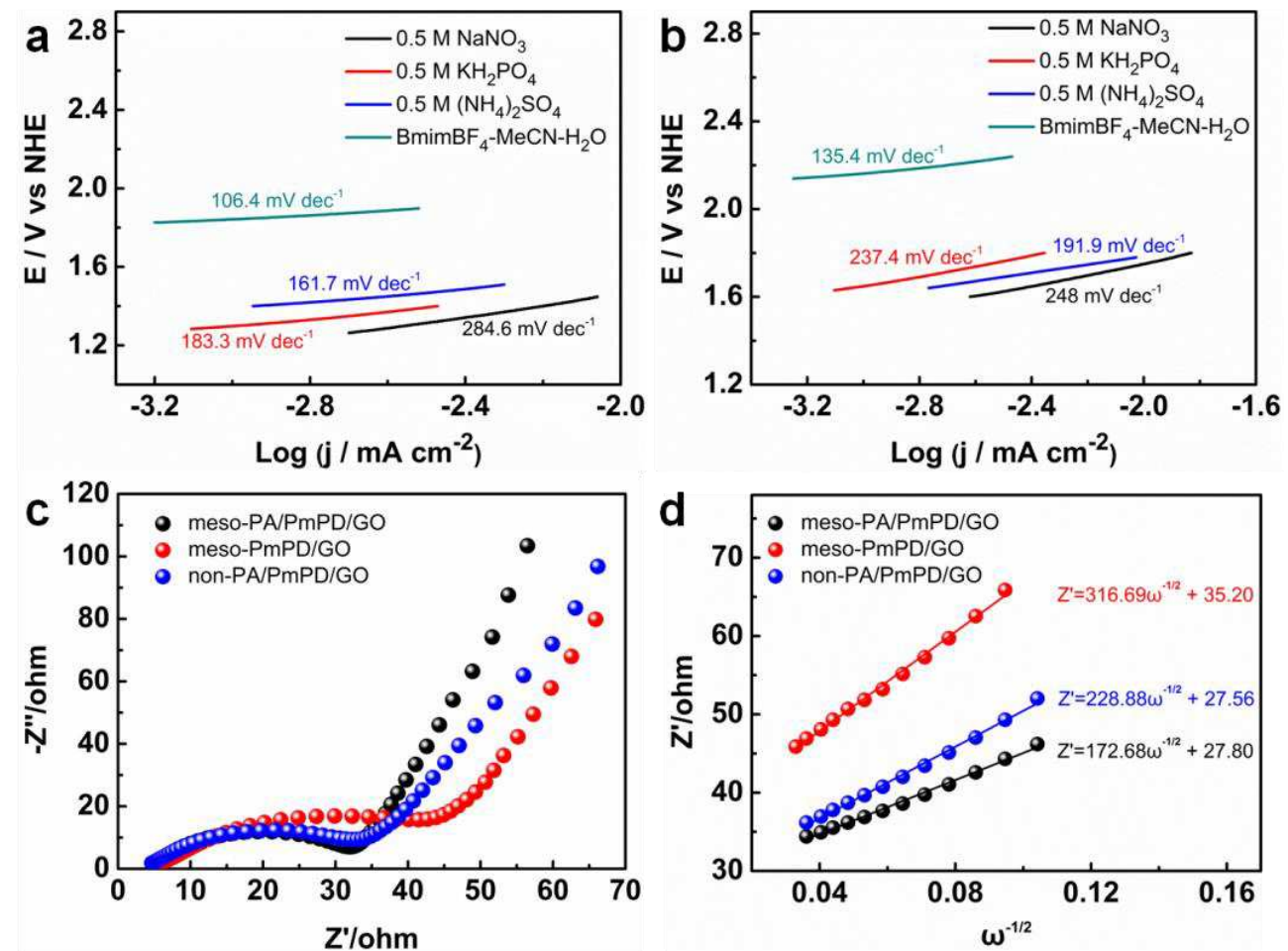

Fig. 7 Tafel plots of different electrolytes over meso-PA/PmPD/GO in the presence of furfuryl alcohol (a) and furfural (b). (c) EIS spectra. (d) Variations and fittings between Z' and the reciprocal square root of the angular frequency $(\omega)$ in the low frequency region of meso-PA/PmPD/GO, meso-PmPD/GO, and non-PA/PmPD/GO in the mixture of furfuryl alcohol and the ternary electrolyte.

The contribution of the PA incorporation to the excellent electrocatalytic performance of meso-PA/PmPD/GO was also studied. The ${ }^{1} \mathrm{H}$ NMR spectra (Supplementary Fig. S24) of the mixture of PA and furfuryl alcohol in DMSO- $d_{6}$ reveal that the chemical shifts $(\mathrm{H}-1, \mathrm{H}-2, \mathrm{H}-5$, and H-6) in furfuryl alcohol exhibit an obvious high field shift, while the chemical shifts of PA move to the low field with an increase in the PA concentration. This phenomenon indicates the presence of hydrogen-bonding interaction between PA and furfuryl alcohol, ${ }^{53}$ which is beneficial to the adsorption of furfuryl alcohol molecules on the surface of meso-PA/PmPD/GO to be catalyzed. A similar phenomenon is also observed in the ${ }^{1} \mathrm{H}$ NMR spectra of the PA and furfural mixture (Supplementary Fig. S25). Moreover, PA, as an active dopant, possesses the ability to improve the 
electrochemical activity of materials. ${ }^{54-57}$ The electrochemical impedance spectra (EIS) give a smaller semicircle radius for meso-PA/PmPD/GO nanosheets than that for meso-PmPD/GO (Fig. 7c and Supplementary Fig. S23a), demonstrating that the doping of PA may decrease the charge transfer resistance at the interface of meso-PA/PmPD/GO and electrolyte, and thus favor the charge transport.

Further, the inverted 'V-shaped' Mott-Schottky (M-S) curve indicates the formation of a p-n heterojunction interface in meso-PA/PmPD/GO (Supplementary Fig. S26a). Fig. S26b in Supplementary verifies that the PA/PmPD layer is an n-type semiconductor in light of the positive slope of the M-S curve, while GO is usually a p-type semiconductor. ${ }^{58}$ The flat band potential $\left(\mathrm{E}_{\mathrm{fb}}\right)$ of PA/PmPD is $-0.42 \mathrm{~V}$ vs NHE, which is determined by the extrapolation of the linear region of the M-S curve (Supplementary Fig. S26b). As the conduction band (CB) value of an n-type semiconductor is around $0.2 \mathrm{~V}$ lower than $\mathrm{E}_{\mathrm{fb}},{ }^{59}$ the $\mathrm{CB}$ value of PA/PmPD is $-0.62 \mathrm{~V}$ vs. NHE. Ultroviolet photoelectron spectrometer (UPS) measurement suggests that the valence band (VB) values of PA/PmPD and GO are 0.59 V and 1.17 V vs NHE, respectively (Supplementary Fig. S27). UV-vis absorption spectra give a bandgap of $4.22 \mathrm{eV}$ for GO (Supplementary Fig. S28). Accordingly, the $\mathrm{CB}$ value of GO is calculated to be $-3.05 \mathrm{~V}$ vs NHE. Thereby, the $\mathrm{CB}$ and VB edges of PA/PmPD are enveloped within the energy gap of GO. Based on these results, a reverse type-I heterojunction mechanism is proposed for the charge separation and transfer at the interface of the meso-PA/PmPD/GO nanosheets during the electrocatalytic oxidations of the furan-based biomass (Fig. 8). Upon electrical excitation, the electrons on the $\mathrm{CB}$ of GO transfer to that of the PA/PmPD layer, while the holes on the VB of GO simultaneously hop to that of PA/PmPD. Due to the difference between the migration rates of electrons and holes, this leads to an efficient separation of the carriers at the interface. ${ }^{60,61}$ In the aforementioned 
$\mathrm{Bmim}^{+}$-to-Bmim*-to-BmimH-to-Bmim ${ }^{+}$cycle, it is known that $\mathrm{Bmim}^{*}$ generated from $\mathrm{Bmim}^{+}$ triggers the oxidation of the furan-based molecules and thus transforms to BmimH. At this moment, the holes accumulated on the VB of the PA/PmPD layers capture the electrons from BmimH and convert it back to $\mathrm{Bmim}^{+}$, thereby promoting the $\mathrm{Bmim}^{+}$-to-Bmim*-to-BmimH-to-Bmim ${ }^{+}$cycle (Fig. 8). In stark contrast, for the meso-PmPD/GO nanosheets without the PA doping, the VB edge of the outer PmPD layers is higher than that of the inner GO (Supplementary Fig. S27c). Therefore, a type-II heterojunction is formed in the meso-PmPD/GO nanosheets, where the generated holes on the outer PmPD layers move to the inner GO layers and thus are shielded by the PmPD layers (Supplementary Fig. S29). This situation is not beneficial to the oxidation of BmimH back to $\mathrm{Bmim}^{+}$. This comparison verifies the remarkable contribution of the PA doping to the formation of the reverse type-I heterojunction in the sandwich-structured meso-PA/PmPD/GO nanosheets. The heterojunction structure enables the effective electron-hole separation and the transfer of the holes to the outer PA/PmPD layers, which favors the consecutive $\mathrm{Bmim}^{+}$-to-Bmim*-to-BmimH-to-Bmim ${ }^{+}$cycles and thus boosts the electrocatalytic performance.

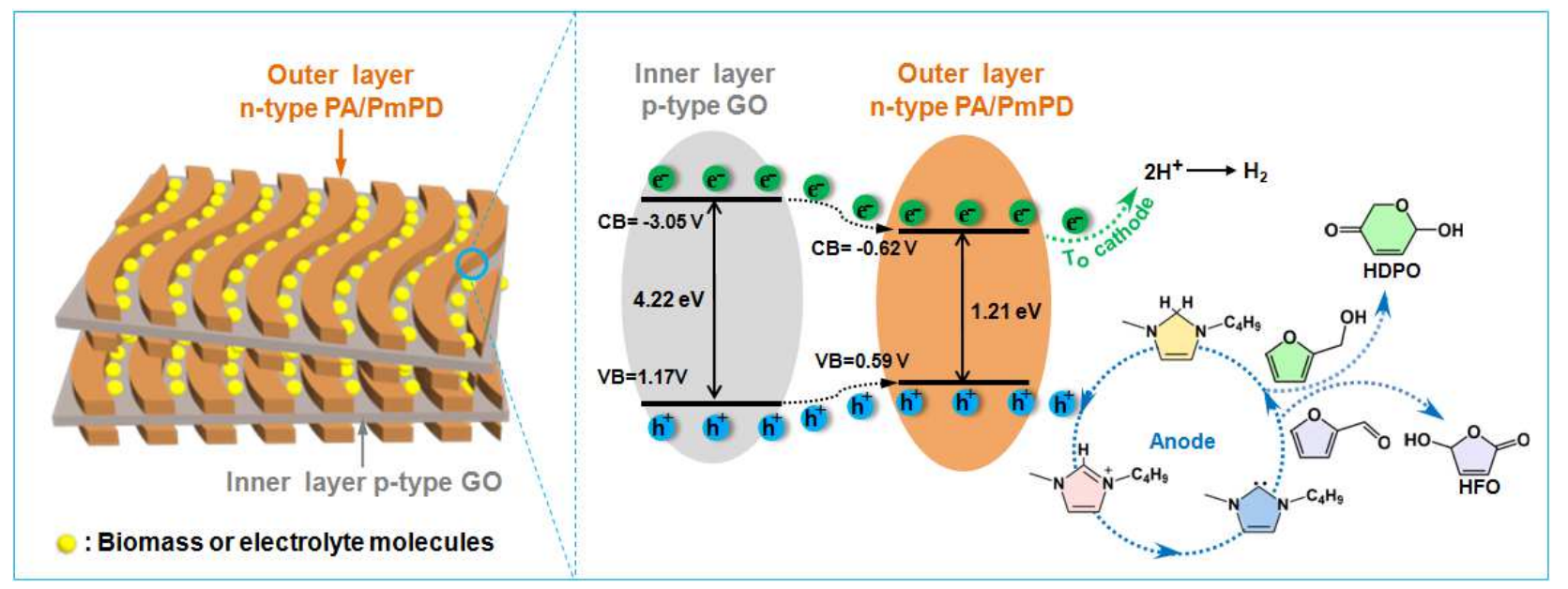

Fig. 8 A possible mechanism of the charge separation and transfer at the p-n heterojunction interface of the meso-PA/PmPD/GO nanosheets during the electrocatalytic oxidation of furfuryl alcohol or furfural. 
In summary, we constructed a phytic-acid-doped 2D metal-free mesoporous electrocatalyst and developed a high-performance ternary electrolyte containing $\mathrm{BmimBF}_{4}$, acetonitrile and $\mathrm{H}_{2} \mathrm{O}$. Using water as the green oxygen source at room temperature, this new electrocatalytic system exhibited excellent catalytic performance for the electrochemical oxidation of furfuryl alcohol to HDPO with high FE (83.7\%) and selectivity (87.9\%), and also ultrahigh activity for the oxidation of furfural to HFO with record-high FE (98.9\%) and selectivity (93.6\%). Mechanism study including DFT calculations unveiled that the $\mathrm{Bmim}^{*}$ originated from $\mathrm{BmimBF}_{4}$ was the reaction-determining active species. Additionally, the synergetic effect of the PA doping, the mesoporous structure, and the $\mathrm{p}$-n heterojunction interface in the $2 \mathrm{D}$ catalyst favored the mass transfer, the electron-hole separation, and the transport of holes to the outer layers, thus optimizing the catalytic performance. This study provides insightful clues to develop more advanced electrocatalytic systems for efficient conversion of biomass to high value-added chemicals.

\section{Methods}

Materials. 1-Butyl-3-methylimidazolium tetrafluoroborate $\left(\mathrm{BmimBF}_{4}, 98 \%\right)$ was purchased from the Centre of Green Chemistry and Catalysis, Lanzhou Institute of Chemical Physics, Chinese Academy of Sciences. Phytic acid (PA, $50 \mathrm{wt} \%$ ), m-phenylenediamine (99\%), and ammonium persulfate (99\%) were supplied by J\&K Scientific Co., Ltd. Poly(ethylene oxide) $)_{20}$-block-poly(phenylene oxide) ${ }_{70}$-block-poly(ethylene oxide) ${ }_{20}(\mathrm{P} 123,99 \%)$, furfuryl alcohol (98\%), furfural (98\%), and 5-methylfurfural (98\%) were obtained from Sinophar Chemical Reagent Co., Ltd. Nafion D-521 dispersion $(5 \% \mathrm{w} / \mathrm{w}$ in water and 1-propanol, $\geq 0.92 \mathrm{meg} / \mathrm{g}$ exchange capacity), Nafion N-117 membrane ( $0.180 \mathrm{~mm}$ thick, $\geq 0.90 \mathrm{meg} / \mathrm{g}$ exchange capacity), and Toray Carbon Paper (CP, TGP-H-60, 19×19 cm) were purchased from Alfa Aesar China Co., Ltd. 
Preparation of 2D mesoporous electrocatalysts. Taking the meso-PA/PmPD/GO as an example, First, GO was synthesized according to the modified Hummers method. ${ }^{62}$ P123 (0.4 g) was dissolved in deionized water $(40 \mathrm{~mL})$. Then, $8 \mathrm{~mL} \mathrm{GO}$ aqueous solution $\left(0.82 \mathrm{mg} \mathrm{mL}^{-1}\right)$ was added into the mixture solution. After mild stirring at $35^{\circ} \mathrm{C}$ for $1 \mathrm{~h}, \mathrm{~m}$-phenylenediamine $(50 \mathrm{mg})$ and PA $(33 \mathrm{uL})$ were added into the solution. The mixture was stirred continuously for $1 \mathrm{~h}$, then $20 \mathrm{~mL}$ aqueous solution of ammonium persulfate $\left(10 \mathrm{mg} \mathrm{mL}^{-1}\right)$ was added dropwise to initiate the cross-linking polymerization of m-phenylenediamine and PA. Afterwards, the resultant black precipitate was washed with ethanol and water to remove the P123 template. The product is denoted as meso-PA/PmPD/GO.

For the synthesis of meso-PmPD/GO, the procedures were similar to the meso-PA/PmPD/GO except the addition of PA. For non-PA/PmPD/GO, the difference is without the addition of P123 as the template in the reaction system.

Preparation of the electrodes. The obtained electrocatalyst $(10.0 \mathrm{mg})$ was suspended in $1 \mathrm{~mL}$ ethanol with $20 \mu \mathrm{L}$ Nafion D-521 dispersion (5 wt\%) to form a homogeneous ink with the assistance of ultrasound. After that, the ink was spread onto carbon paper $(\mathrm{CP})(1 \mathrm{~cm} \times 1 \mathrm{~cm})$. The $\mathrm{CP}$ was pretreated by the calcination at $500{ }^{\circ} \mathrm{C}$ in the air before use. Finally, the electrode was dried under room temperature.

Characterizations. Transmission electron microscope (TEM, JEOL-2100) and field emission transmission electron microscope (FETEM, TALOS F200X) with an accelerating voltage of $200 \mathrm{kV}$ were used for the TEM characterizations. TEM samples were prepared by dropping a drop of the sample dispersion containing ethanol on copper grids, followed by air drying at room temperature. Scanning electron microscopy (SEM) experiment was conducted on a Hitachi S-4800 scanning electron microscope operated at $15 \mathrm{kV}$. SEM samples were prepared by dropping a drop of the 
sample dispersion containing ethanol on silicon wafers, followed by air drying at room temperature. Powder X-ray diffraction (XRD) patterns were collected on a Rigaku D/max-2500 X-ray diffractometer using $\mathrm{Cu} \mathrm{K} \alpha$ radiation $(\lambda=0.154 \mathrm{~nm})$. Ultraviolet photoelectron spectroscopy (UPS) was conducted on the Scienta R3000 spectrometer system using using HeI $21.22 \mathrm{eV}$ as exciting source with energy resolution of $50 \mathrm{meV} .{ }^{1} \mathrm{H}$ NMR spectra were recorded on a Bruker Avance III HD $400 \mathrm{MHz}$ NMR spectrometer. Fourier transform infrared spectra (FTIR) were conducted using a Nicolet 6700. UV-Vis spectra were carried out on a UV/EV300 spectrophotometer (Thermo Fisher). Atomic Force Microscopy (AFM) images were collected on Nanonavi E-Sweep (Japan). Raman spectroscopy was conducted on a Senterra R200-L (Bruker Optics). Gas chromatography-mass spectrometer (GC-MS) measurement (Agilent 5975C-7890A) was used for the qualitative analysis of products.

Linear sweep voltammetry (LSV) measurements. An electrochemical workstation (CHI 660E, Shanghai CH Instruments Co., China) was employed in all the electrochemical experiments in this work. Linear sweep voltammetry (LSV) measurements were conducted on an H-type cell, which was separated by Nafion 117 membrane. A three-electrode system including working electrode, a platinum gauze auxiliary electrode, and a $\mathrm{Ag} / \mathrm{Ag}^{+}\left(0.01 \mathrm{M} \mathrm{AgNO}_{3}\right.$ in $0.1 \mathrm{M}$ TBAP-MeCN) reference electrode was adopted. The reference of $\mathrm{Ag}_{/} \mathrm{Ag}^{+}$is converted to $\mathrm{NHE}$ in all electrochemical experiments. Prior to experiment, the air in the electrolyte was removed by bubbling with $\mathrm{N}_{2}$ for 30 minutes.

Electrochemical impedance spectroscopy (EIS). EIS was conducted in a single compartment cell with three electrodes, including a working electrode, a platinum gauze auxiliary electrode, and a $\mathrm{Ag} / \mathrm{Ag}^{+}(0.01 \mathrm{M} \mathrm{AgNO} 3$ in $0.1 \mathrm{M}$ TBAP-MeCN) reference electrode. The EIS spectra were 
collected using the potentiostatic mode at an open circuit potential of $100 \mathrm{kHz}$ to $100 \mathrm{mHz}$ with an amplitude of $5 \mathrm{mV}$.

Electrochemical oxidations of furfuryl alcohol and furfural. Electrochemical oxidations of furfuryl alcohol and furfural were performed in a typical H-type cell at room temperature. The anodic and cathodic electrolytes were $\mathrm{BmimBF}_{4}-\mathrm{MeCN}-\mathrm{H}_{2} \mathrm{O}$ and aqueous $\mathrm{H}_{2} \mathrm{SO}_{4}$ solution (0.5 M), respectively. The amount of electrolyte in each chamber was $5.9 \mathrm{~g}$ in all experiments. Prior to electrolysis, the anolyte was bubbled with $\mathrm{N}_{2}$ gas for 30 min under stirring. Then, furfuryl alcohol $(0.45 \mathrm{mmol})$ or furfural $(0.5 \mathrm{mmol})$ was added into the anolyte. The electrochemical reaction was started at a desired applied potential for $2 \mathrm{~h}$. The liquid product was analyzed by ${ }^{1} \mathrm{H}$ NMR (Bruker Avance III $400 \mathrm{HD}$ spectrometer) in DMSO- $d_{6}$ or $\mathrm{D}_{2} \mathrm{O}$. The conversion and selectivity of the reaction were calculated in terms of ${ }^{1} \mathrm{H}$ NMR spectra.

Density functional theory (DFT) calculations. DFT calculations were carried out using the Gaussian 09 program. ${ }^{43}$ Geometric optimization, transition state searches, vibrational analysis and a series of single point energy were performed at the B3LYP $\mathrm{L}^{63-65} / 6-311 \mathrm{G}(\mathrm{d}, \mathrm{p})$ level. In this work, structures of transition states were determined by relaxed PES scans and transition state searches, then confirmed using frequency calculations and IRC calculations. Solvation Model Based on Density (SMD) ${ }^{66}$ were used in all calculations involved in this work to provide the effect of implicit solvent (acetonitrile). Unless otherwise specified, all discussed energies were Gibbs free energies. The corresponding coordinates of DFT-computed stationary points were shown in Page S24-S42.

\section{Acknowledgements}

The authors are grateful for Prof. Alberto Bianco at University of Strasbourg (France) for discussion and suggestions. The authors appreciate the financial support from National Natural Science 
Foundation of China (21774076, 52073173 and 21875067), Program of Shanghai Academic Research Leader (19XD1421700) and Program of Distinguished Professor of Special Appointment at Shanghai Institutions of Higher Learning. The Instrumental Analysis Center of Shanghai Jiao Tong University is also acknowledged for some measurements.

\section{Author contributions}

H.W. and Y.M. proposed the project, designed and conducted the experiments and wrote the manuscript. H.X. drew some schematic diagrams. Y.Z. and L.W. conducted the DFT calculations. J.Y. and Q.B. performed some characterizations of the samples. All the authors discussed the results and wrote the manuscript.

\section{Competing interests}

The authors declare no competing financial interests.

\section{References}

1. Liguori, F., Moreno-Marrodan, C. \& Barbaro, P. Biomass-derived chemical substitutes for bisphenol A: recent advancements in catalytic synthesis. Chem. Soc. Rev. 49, 6329-6363 (2020).

2. Wong, S. S., Shu, R., Zhang, J., Liu, H. \& Yan, N. Downstream processing of lignin derived feedstock into end products. Chem. Soc. Rev. 49, 5510-5560 (2020).

3. Mika, L. T., Cséfalvay, E. \& Németh, Á. Catalytic conversion of carbohydrates to initial platform chemicals: chemistry and sustainability. Chem. Rev. 118, 505-613 (2018).

4. Schutyser, W. et al. Chemicals from lignin: an interplay of lignocellulose fractionation, depolymerisation, and upgrading. Chem. Soc. Rev. 47, 852-908 (2018).

5. Li, S. et al. Selective hydrogenation of 5-(hydroxymethyl) furfural to 5-methylfurfural over single atomic metals anchored on $\mathrm{Nb}_{2} \mathrm{O}_{5}$. Nat. Commun. 12, 584 (2021). 
6. Zhang, Z., Song, J. \& Han, B. Catalytic transformation of lignocellulose into chemicals and fuel products in ionic liquids. Chem. Rev. 117, 6834-6880 (2017).

7. Liu, W.-J. et al. Efficient electrochemical production of glucaric acid and $\mathrm{H}_{2}$ via glucose electrolysis. Nat. Commun. 11, 265 (2020).

8. Lu, Y. et al. Identifying the geometric site dependence of spinel oxides for the electrooxidation of 5-hydroxymethylfurfural. Angew. Chem. Int. Ed. 59, 19215- 19221 (2020).

9. Poerwoprajitno, A. R. et al. Faceted branched nickel nanoparticles with tunable branch length for high-activity electrocatalytic oxidation of biomass. Angew. Chem. Int. Ed. 59, 15487-15491 (2020). 10. Zhang, P. et al. Paired electrocatalytic oxygenation and hydrogenation of organic substrates with water as the oxygen and hydrogen source. Angew. Chem. Int. Ed. 58, 9155-9159 (2019).

11. You, B., Jiang, N., Liu, X. \& Sun, Y. Simultaneous $\mathrm{H}_{2}$ generation and biomass upgrading in water by an efficient noble-metal-free bifunctional electrocatalyst. Angew. Chem. Int. Ed. 55, 9913-9917 (2016).

12. Barwe, S. et al. Electrocatalytic oxidation of 5-(hydroxymethyl)furfural using high-surface-area Nickel Boride. Angew. Chem. Int. Ed. 57, 11460-11464 (2018).

13. Zhou, C. et al. Oxidation of 5-hydroxymethylfurfural over a magnetic iron oxide decorated rGO supporting Pt nanocatalyst. Catal. Today 330, 92-100 (2019).

14. Geneste, F. \& Moinet, C. Electrocatalytic oxidation of alcohols by a $\left[\mathrm{Ru}(\mathrm{tpy})(\mathrm{phen})\left(\mathrm{OH}_{2}\right)\right]^{2+}$-modified electrode. J. Electroanal. Chem. 594, 105-110 (2006).

15. Bosco, J. A. et al. Redox-mediated oxidation of alcohols using $\mathrm{Cl}^{-} / \mathrm{OCl}^{-}$redox couple in biphasic media. J. Phys. Org. Chem. 28, 591-595 (2015).

16. Orue, A., Uria, U., Reyes, E., Carrillo, L. \& Vicario, J. L. Catalytic enantioselective [5+2] cycloaddition between oxidopyrylium ylides and enals under dienamine activation. Angew. Chem. 
Int. Ed. 54, 3043-3046 (2015).

17. Suga, H., Iwai, T., Shimizu, M., Takahashi, K. \& Toda, Y. Efficient generation of an oxidopyrylium ylide using a Pd catalyst and its [5+2] cycloadditions with several dipolarophiles. Chem. Commun. 54, 1109-1112 (2018).

18. Zhao, C., Li, F. \& Wang, J. N-heterocyclic carbene catalyzed dynamic kinetic resolution of pyranones. Angew. Chem. Int. Ed. 55, 1820-1824 (2016).

19. Kuo, Y. -H. \& Shih, K.-S. Preparation of 6-hydroxy-2H-pyran-3(6H)-one from 2-furylcarbinol by photooxidation. synthesis of a pheromone of vespa orientalis. Heterocycles 31, 1941-1949 (1990).

20. Falenczyk, C., Pölloth, B., Hilgers, P. \& König, B. Mechanochemically initiated achmatowicz rearrangement. Synthetic Commun. 45, 348-354 (2015).

21. Liu, X. et al. Electrocatalytic synthesis of heterocycles from biomass-derived furfuryl alcohols. Nat. Commun. 12, 1868 (2021).

22. Kumar, P. \& Pandey, R. K. An efficient synthesis of 5-hydroxy-2(5H)-furanone using a titanium silicate molecular sieve catalyst. Green Chem. 2, 29-32 (2000).

23. Heidary, N. \& Kornienko, N. Operando vibrational spectroscopy for electrochemical biomass valorization. Chem. Commun. 56, 8726-8734 (2020).

24. $\mathrm{Wu}, \mathrm{H}$. et al. An electrocatalytic route for transformation of biomass-derived furfural into 5-hydroxy-2(5H)-furanone. Chem. Sci. 10, $4692-4698$ (2019).

25. Thiyagarajan, S. et al. Selective production of maleic acid from furfural via a cascade approach combining photochemistry and electro- or biochemistry. ACS Sustainable Chem. Eng. 8, 10626-10632 (2020).

26. Román, A. M., Hasse, J. C., Medlin, J. W. \& Holewinski, A. Elucidating acidic electro-oxidation 
pathways of furfural on platinum. ACS Catal. 9, 10305-10316 (2019).

27. Wang, J. et al. Hierarchical porous carbons with layer-by-layer motif architectures from confined soft-template self-assembly in layered materials. Nat. Commun. 8, 15717 (2017).

28. Hou, D. et al. Two-dimensional sandwich-structured mesoporous Mo2C/carbon/graphene nanohybrids for efficient hydrogen production electrocatalysts. ACS Appl. Mater. Interfaces 10, 40800-40807 (2018)

29. Shi, H. et al. A two-dimensional mesoporous polypyrrole-graphene oxide heterostructure as a dual-functional ion redistributor for dendrite-free lithium metal anodes. Angew. Chem. Int. Ed. 59, 12147-12153 (2020).

30. Zhu, Q. et al. Efficient reduction of $\mathrm{CO}_{2}$ into formic acid on a lead or tin electrode using an ionic liquid catholyte mixture. Angew.Chem. Int.Ed. 55, 9012-9016 (2016).

31. Wang, T., Okejiri, F., Qiao, Z.-A. \& Dai, S. Tailoring polymer colloids derived porous carbon spheres based on specific chemical reactions. Adv. Mater. 32, 2002475 (2020).

32. Li, C. et al. Self-assembly of block copolymers towards mesoporous materials for energy storage and conversion systems. Chem. Soc. Rev. 49, 4681-4736 (2020).

33. Sun, H. et al. Template-free fabrication of nitrogen-doped hollow carbon spheres for high-performance supercapacitors based on a scalable homopolymer vesicle. J. Mater. Chem. A 4, 12088-12097 (2016).

34. Deng, Y., Wei, J., Sun, Z. \& Zhao, D. Large-pore ordered mesoporous materials templated from non-pluronic amphiphilic block copolymers. Chem. Soc. Rev. 42, 4054-4070 (2013).

35. Wang, J. et al. Universal access to two-dimensional mesoporous heterostructures by micelle-directed interfacial assembly. Angew. Chem. Int. Ed. 59, 19570-19575 (2020).

36. Li, Q. et al. Ordered bicontinuous mesoporous polymeric semiconductor photocatalyst. ACS 
Nano 14, 13652-13662 (2020).

37. Guo, B. et al. Controlled synthesis of porous carbon nanostructures with tunable closed mesopores via a silica-assisted coassembly strategy. CCS Chem. 2, 1410-1422 (2020).

38. Alexandridis, P., Holzwarth, J. F. \& Hatton, T. A. Micellization of poly(ethylene oxide)-poly(propylene oxide)-poly(ethylene oxide) triblock copolymers in aqueous solutions: thermodynamics of copolymer association. Macromolecules 27, 2414-2425 (1994).

39. Tian, H. et al. General interfacial self-assembly engineering for patterning two dimensional polymers with cylindrical mesopores on graphene. Angew. Chem. Int. Ed. 58, 10173-10178 (2019). 40. Liu, S. et al. Dual-template synthesis of 2D mesoporous polypyrrole nanosheets with controlled pore size. Adv. Mater. 28, 8365-8370 (2016).

41. Liang, C. \& Dai, S. Synthesis of mesoporous carbon materials via enhanced hydrogen-bonding interaction, J. Am. Chem. Soc. 128, 5316-5317 (2006).

42. Nordness, O. \& Brennecke, J. F. Ion dissociation in ionic liquids and ionic liquid solutions. Chem. Rev. 120, 12873-12902 (2020).

43. Frisch, M. J. et al. Gaussian 09, Revision A.1. Gaussian Inc., Wallingford, CT, (2009).

44. Fèvre, M. et al. Imidazol(in)ium hydrogen carbonates as a genuine source of N-heterocyclic carbenes (NHCs): applications to the facile preparation of NHC metal complexes and to NHC-organocatalyzed molecular and macromolecular syntheses. J. Am. Chem. Soc. 134, 6776-6784 (2012).

45. Gurau, G. et al. Demonstration of chemisorption of carbon dioxide in 1,3-dialkylimidazolium acetate ionic liquids. Angew. Chem. Int. Ed. 50, 12024-12026 (2011).

46. Liu, M. et al. Transformation of alcohols to esters promoted by hydrogen bonds using oxygen as the oxidant under metal-free conditions. Sci. Adv. 4, eaas9319 (2018). 
47. Cotillas, S. et al. Removal of procion red MX-5B dye from wastewater by conductive-diamond electrochemical oxidation. Electrochim. Acta 263, 1-7 (2018).

48. Hamad, H., Bassyouni, D., El-Ashtoukhy, E., Amin, N. \& El-Latif, M. A. Electrocatalytic degradation and minimization of specific energy consumption of synthetic Azo dye from wastewater by anodic oxidation process with an emphasis on enhancing economic efficiency and reaction mechanism. Ecotoxicol. Environ. Saf. 148, 501-512 (2018).

49. Li, X., Ho, B., Lim, D. S. W. \& Zhang Y. Highly efficient formic acid-mediated oxidation of renewable furfural to maleic acid with $\mathrm{H}_{2} \mathrm{O}_{2}$. Green Chem. 19, 914-918 (2017).

50. Su, B.-M., Zhang, S. \& Zhang, Z. C. Structural elucidation of thiophene interaction with ionic liquids by multinuclear NMR spectroscopy. J. Phys. Chem. B 108, 19510-19517 (2004).

51. Zhang, X. et al. Simultaneously high-rate furfural hydrogenation and oxidation upgrading on nanostructured transition metal phosphides through electrocatalytic conversion at ambient conditions. Appl. Catal. B: Environ. 244, 899-908 (2019).

52. Tao, H. B. et al. Revealing energetics of surface oxygen redox from kinetic fingerprint in oxygen electrocatalysis. J. Am. Chem. Soc. 141, 13803-13811 (2019).

53. Zhao, X. et al. Hydrazide-based quadruply hydrogen-bonded heterodimers. structure, assembling selectivity, and supramolecular substitution. J. Am. Chem. Soc. 125, 15128-15139 (2003).

54. Wu, H. et al. Design of naturally derived lead phytate as an electrocatalyst for highly efficient $\mathrm{CO}_{2}$ reduction to formic acid. Green Chem. 20, 4602-4606 (2018).

55. Zhang, G. et al. Highly active and stable catalysts of phytic acid-derivative transition metal phosphides for full water splitting. J. Am. Chem. Soc. 138, 14686-14693 (2016).

56. Zhang, J., Zhao, Z., Xia, Z. \& Dai, L. A metal-free bifunctional electrocatalyst for oxygen 
reduction and oxygen evolution reactions. Nat. Nanotechnol. 10, 444-452 (2015).

57. Tao, S. \& Jiang, D. Covalent organic frameworks for energy conversions: current status, challenges, and perspectives. CCS Chem. 2, 2003-2024 (2020).

58. Yeh, T.-F., Cihlár̆, J., Chang, C.-Y., Cheng, C. \& Teng, H. Roles of graphene oxide in photocatalytic water splitting. Mater. Today 16, 78-84 (2013).

59. Li, X. et al. Engineering heterogeneous semiconductors for solar water splitting. J. Mater. Chem. A 3, 2485-2534 (2015).

60. Li, H., Zhou, Y., Tu, W., Ye, J. \& Zou, Z. State-of-the-art progress in diverse heterostructured photocatalysts toward promoting photocatalytic performance. Adv. Funct. Mater. 25, 998-1013 (2015).

61. Samadi-Maybodi, A. \& Shariati, M. R. A study on the transfer of photo-excited charge carriers within direct and inverted type-I heterojunctions of $\mathrm{CdS}$ and $\mathrm{ZnS}$ QDs. New J. Chem. 42, 9808-9818 (2018).

62. Marcano, D. C. et al. Improved synthesis of graphene oxide. ACS Nano 4, 4806-4814 (2010).

63. Lee, C., Yang, W. \& Parr, R. G. Development of the colle-salvetti correlation-energy formula into a functional of the electron density. Phys. Rev. B: Condens. Matter Mater. Phys. 37, 785-789 (1988).

64. Miehlich, B., Savin, A., Stoll, H. \& Preuss, H. Results obtained with the correlation energy density functionals of becke and Lee, Yang and Parr. Chem. Phys. Lett. 157, 200-206 (1989).

65. Stephens, P. J., Devlin, F. J., Chabalowski, C. F. \& Frisch, M. J. Ab initio calculation of vibrational absorption and circular dichroism spectra using density functional force fields. J. Phys.

Chem. 98, 11623-11627 (1994).

66. Marenich, A. V., Cramer, C. J. \& Truhlar, D. G. Universal solvation model based on solute 
electron density and on a continuum model of the solvent defined by the bulk dielectric constant and atomic surface tensions. J. Phys. Chem. B. 113, 6378-6396 (2009). 


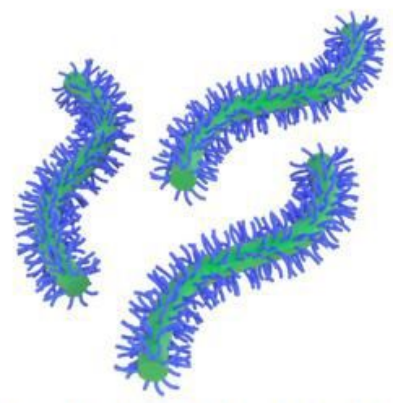

P123 cylindrical micelles

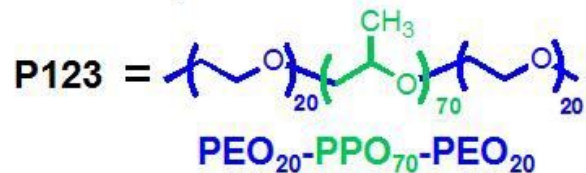

Graphene oxide

Interfacial self-assembly



\section{Precursor monomer}
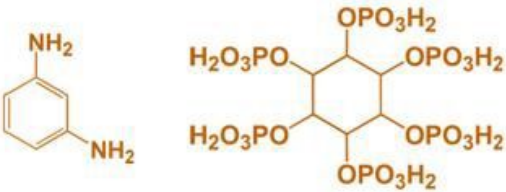

$\mathrm{PA}$ and $\mathrm{MPD}$ in PEO domain

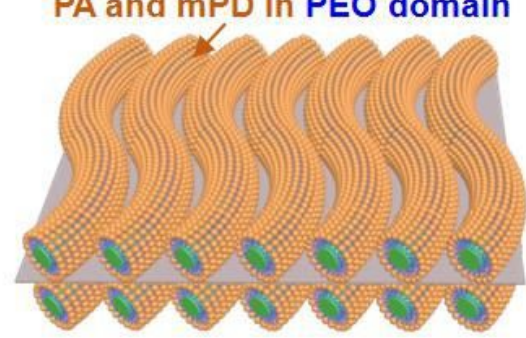

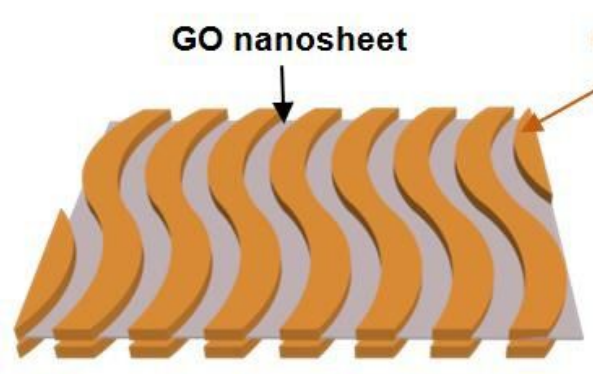

meso-PA/PmPD/Go

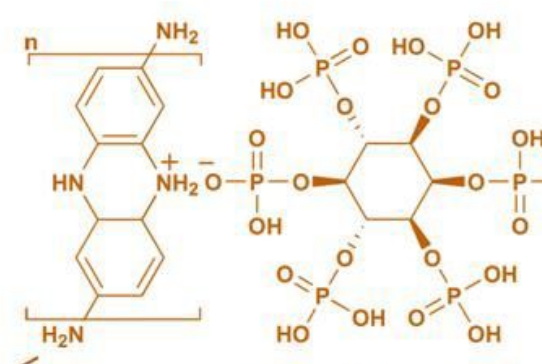

PA/PmPD network

\section{Removal of templates}

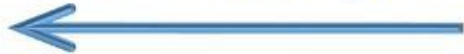

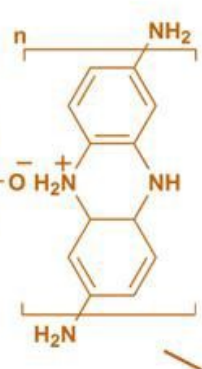

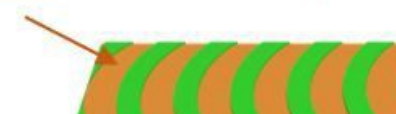



Figure 1

Schematic illustration of the synthesis of meso-PA/PmPD/GO nanosheets with in-plane cylindrical mesopores through an interfacial self-assembly strategy. 

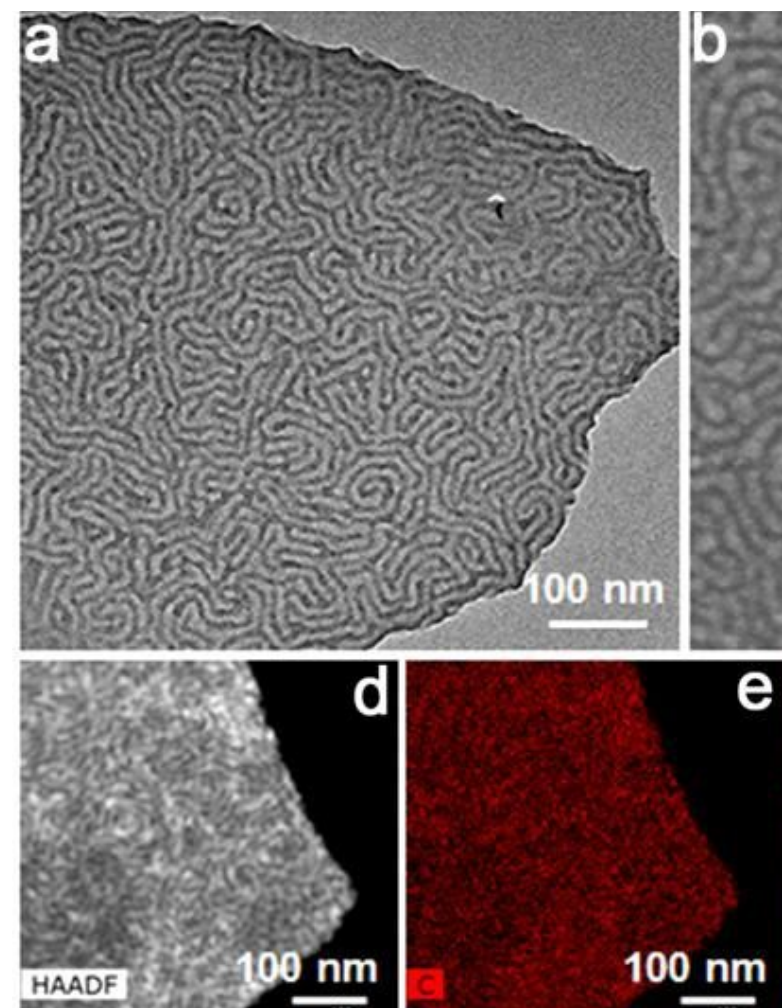
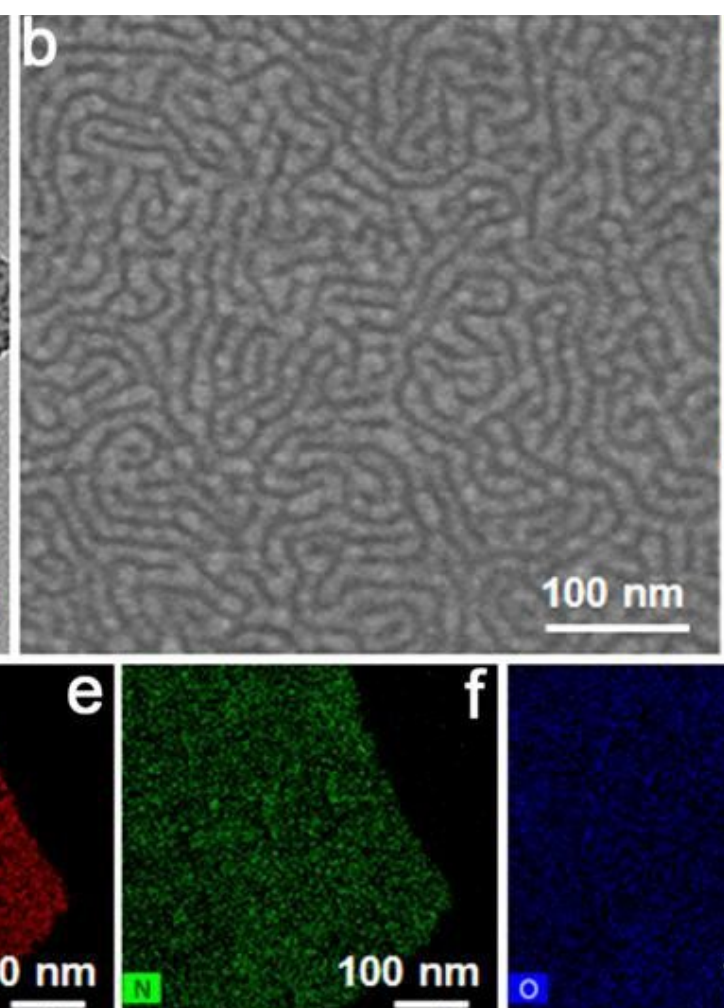
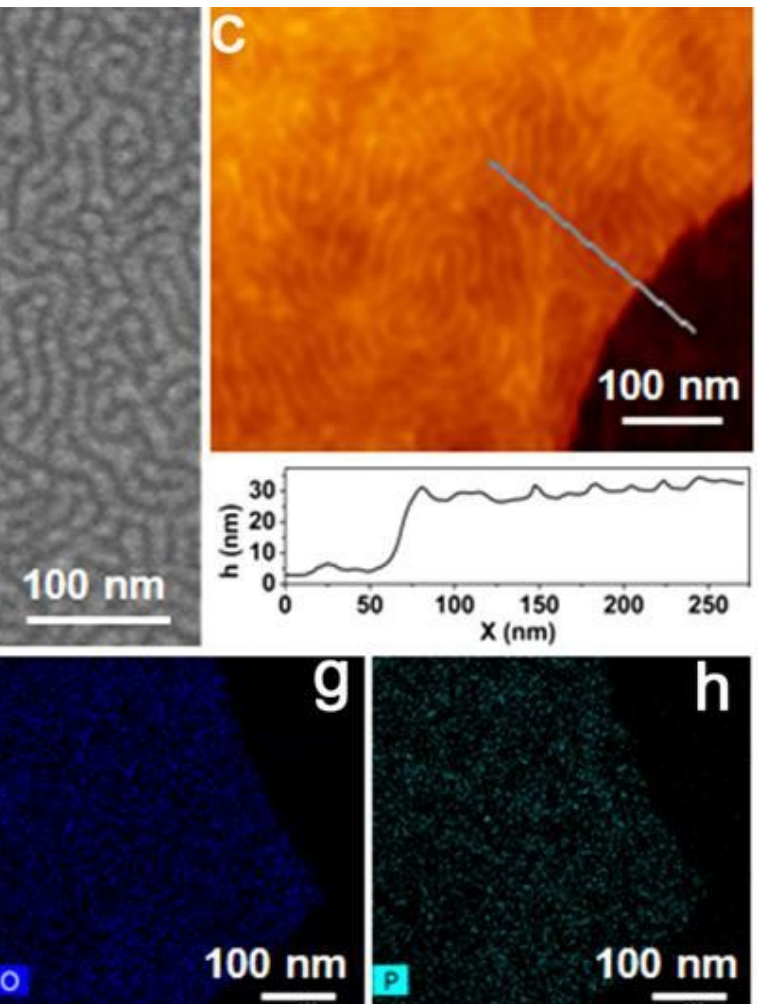

Figure 2

Structural characterizations of the meso-PA/PmPD/GO nanosheets. (a) TEM image; (b) SEM image; (c) AFM image; (d-h) element mapping images. 

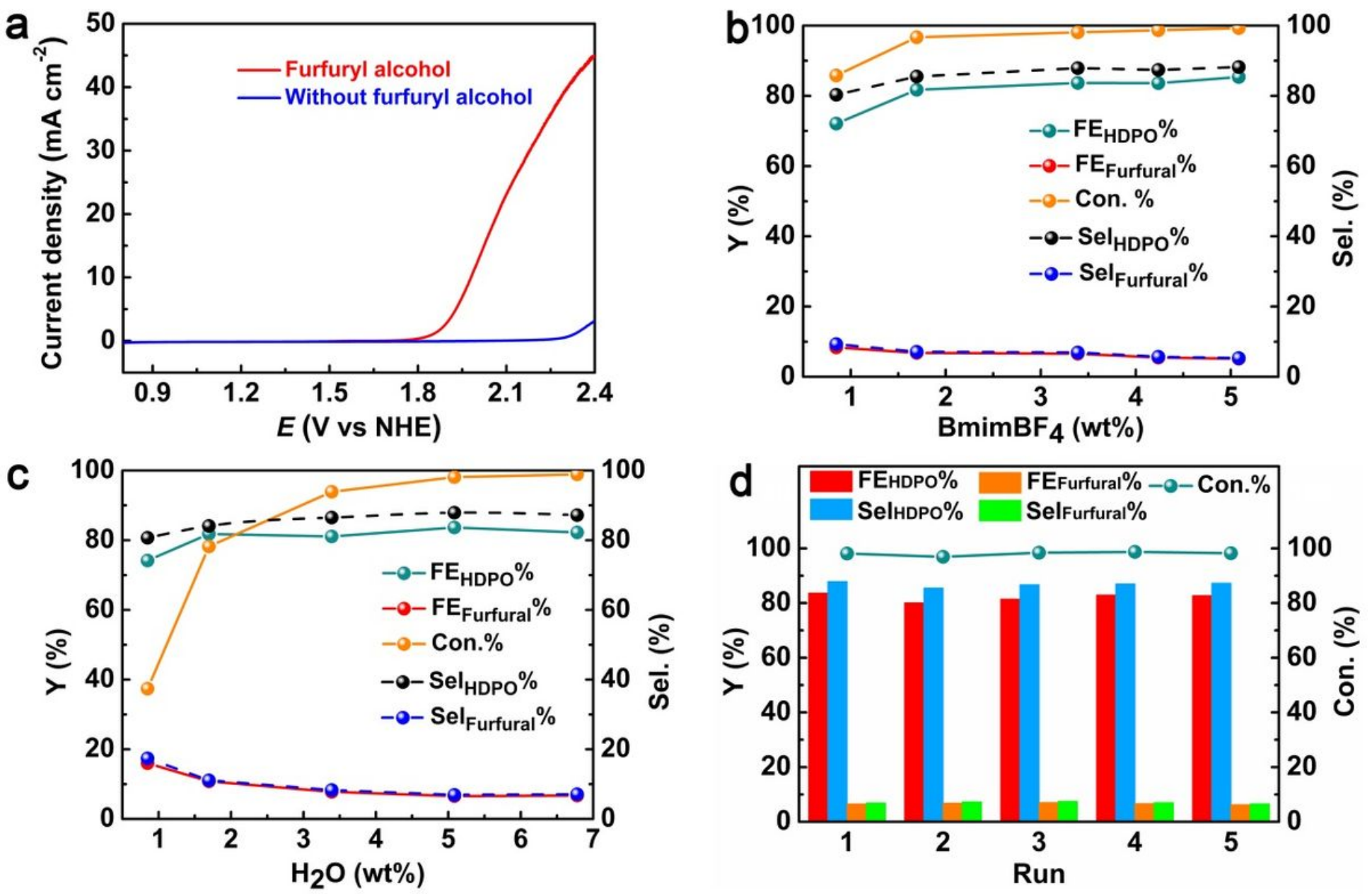

Figure 3

Effect of various parameters on the electrochemical oxidation of furfuryl acohol to HDPO over the mesoPmPD/PA/GO catalyst. (a) LSV measurement; (b) BmimBF4 content; (c) H2O content; (d) cycling performance.
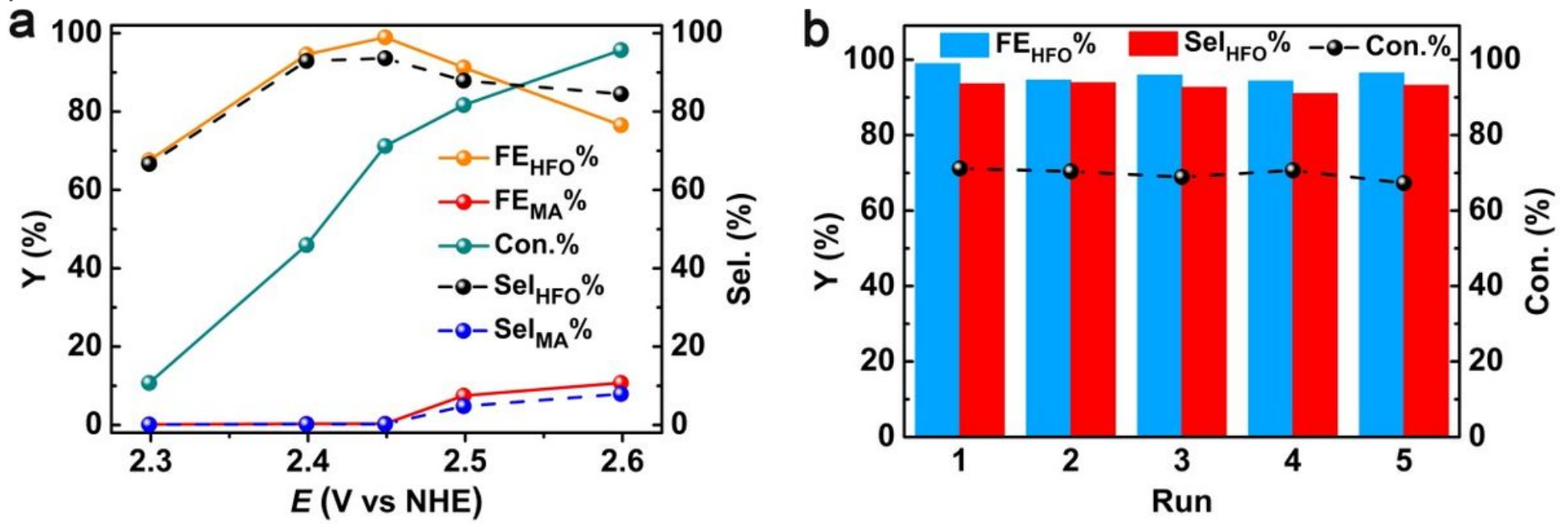

Figure 4 
The electrochemical oxidation of furfural over the meso-PA/PmPD/GO catalyst in the ternary electrolyte. (a) Applied potential and (b) cycling performance.

a<smiles>OCc1ccco1</smiles>
(Furfuryl alcohol)

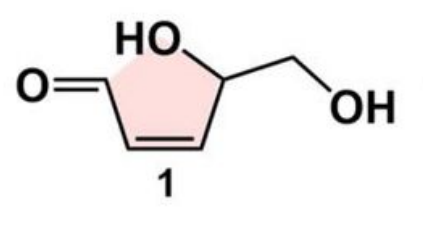

Aldol condensation



(HDPO)

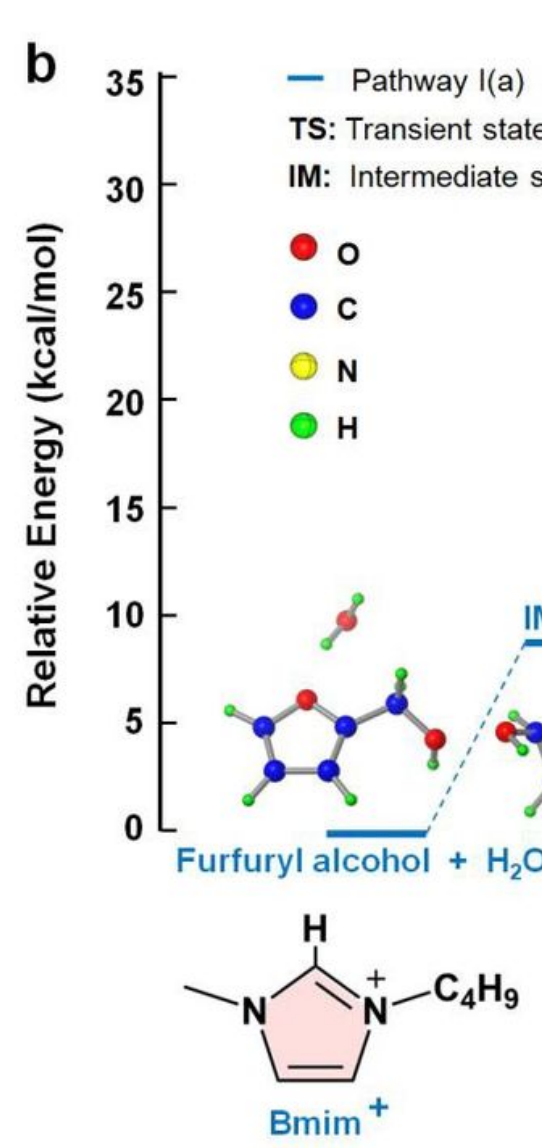
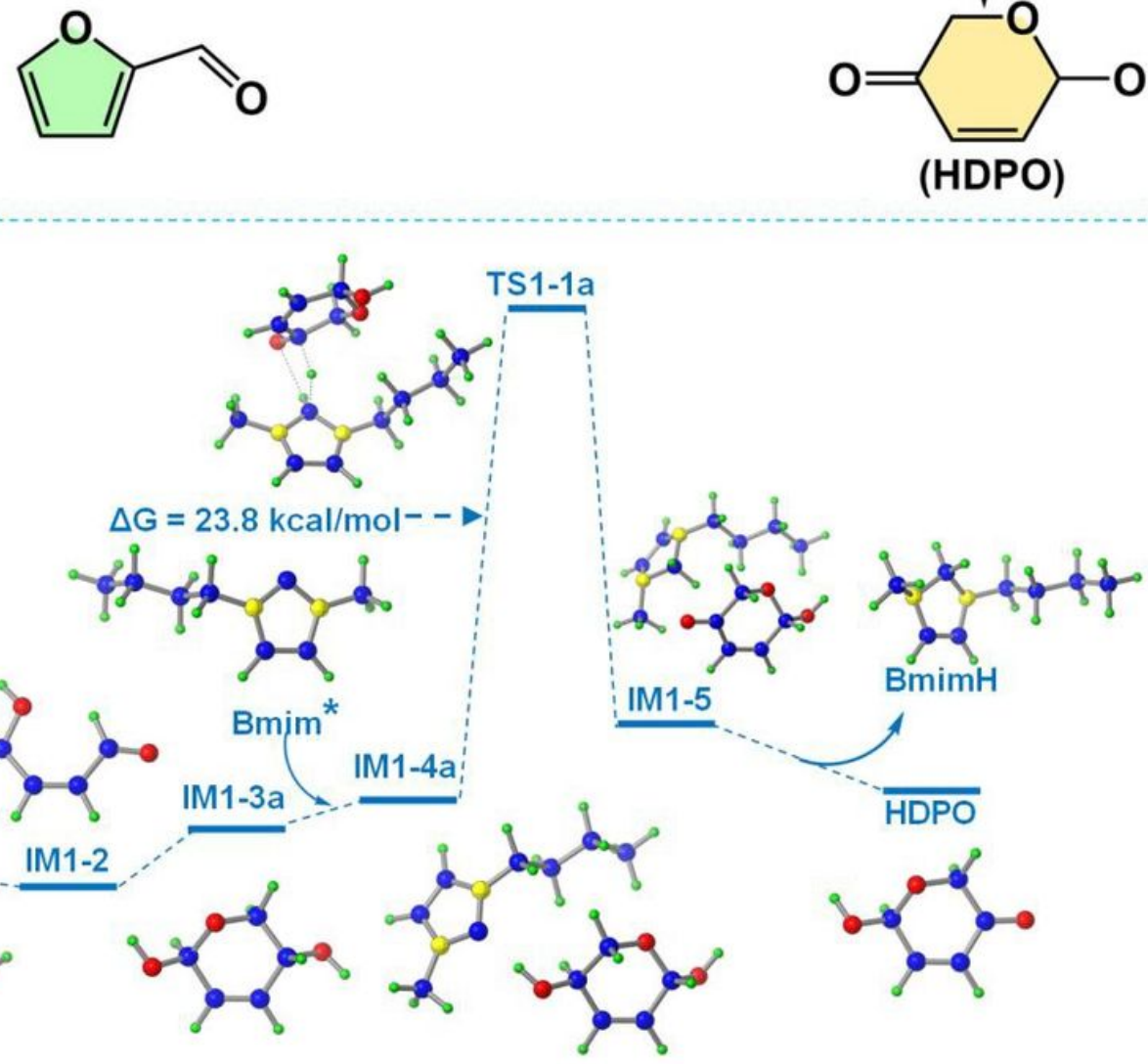
M1-1a $\ldots . . . .1 \mathrm{I} 1-2$


Furfuryl alcohol $+\mathrm{H}_{2} \mathrm{O}$<smiles></smiles>

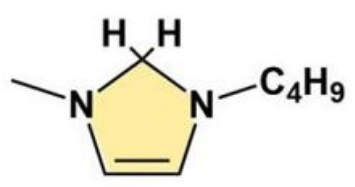

$\mathrm{BmimH}$

Figure 5

(a) Reaction pathways for the electrochemical oxidation of furfuryl alcohol over the meso-PA/PmPD/GO electrocatalyst in the ternary electrolyte. (b) The corresponding DFT calculations. 
a<smiles>O=Cc1ccco1</smiles>

(Furfural)

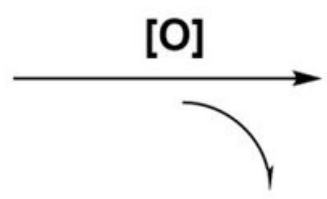

$\mathrm{HCOOH}$



Isomerization

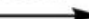<smiles>O=C1CC=CO1</smiles>

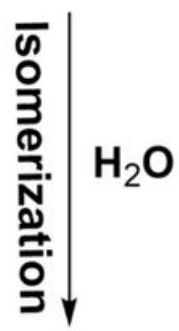<smiles>O=C1C=CC(O)O1</smiles><smiles>C[As](C)(O)[C@H](O)C(O)O</smiles>

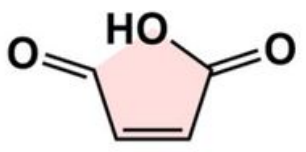

6
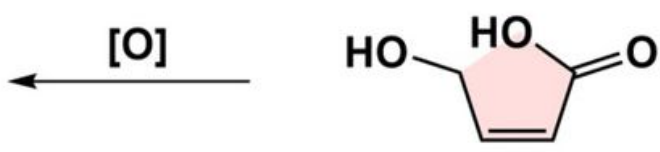

5

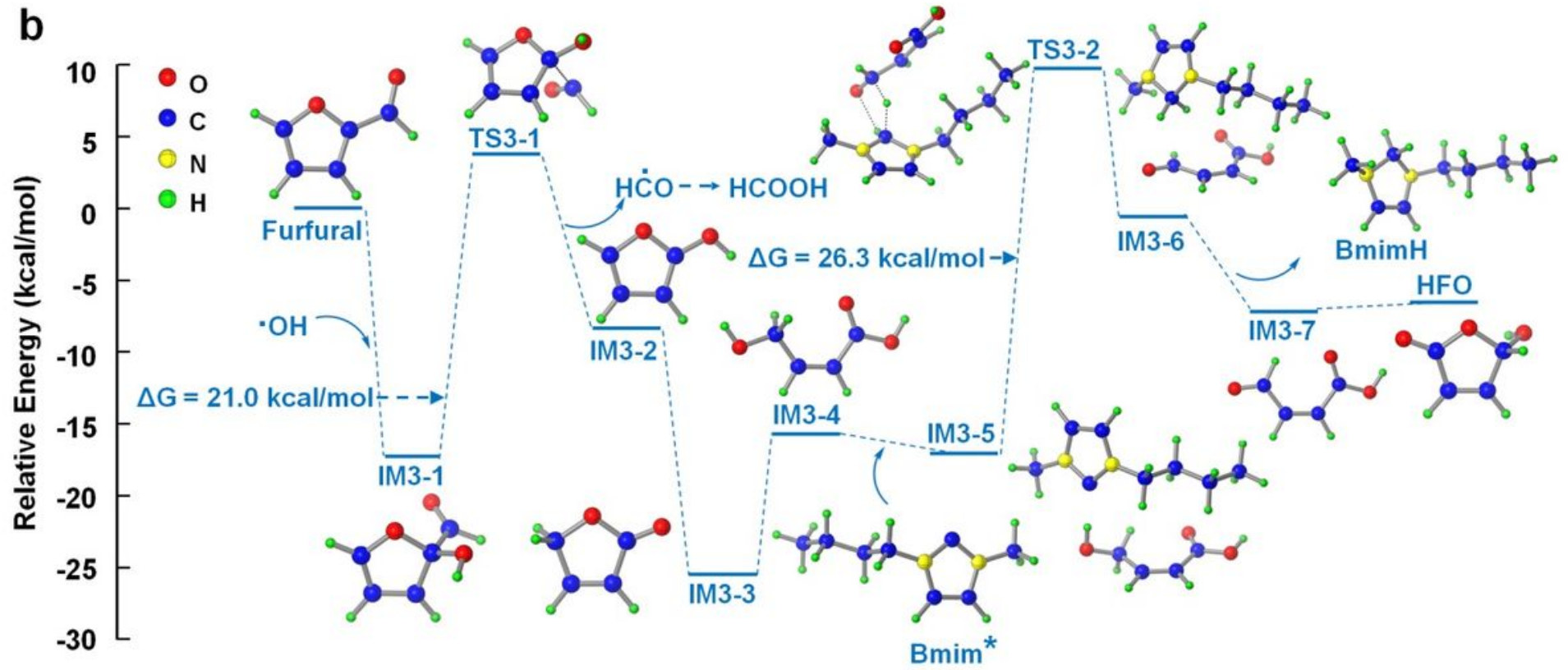

Figure 6

(a) Reaction pathways for the electrochemical oxidation of furfural over the meso-PA/PmPD/GO electrocatalyst in the ternary electrolyte. (b) The corresponding DFT calculations. 

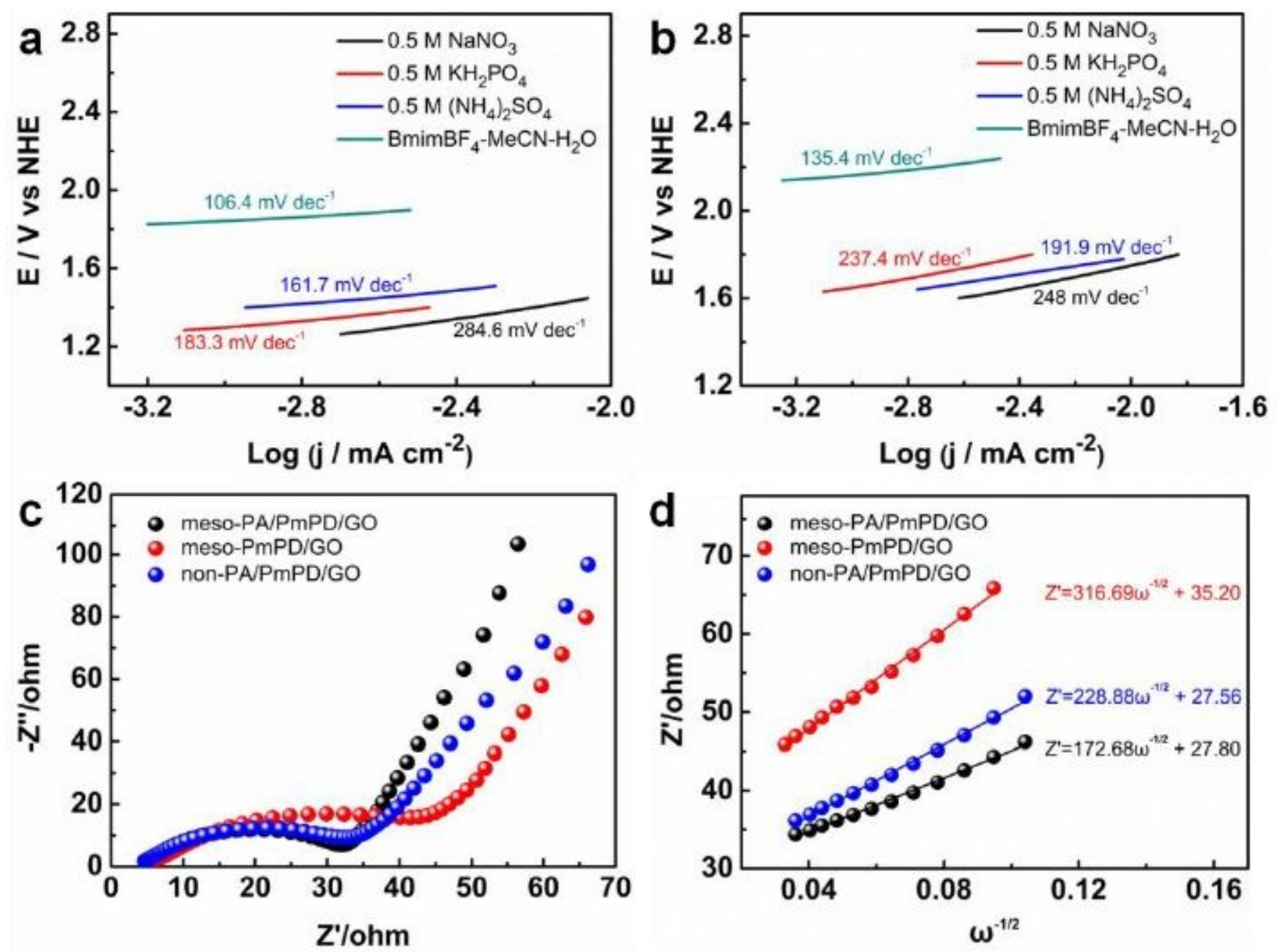

Figure 7

Tafel plots of different electrolytes over meso-PA/PmPD/GO in the presence of furfuryl alcohol (a) and furfural (b). (c) EIS spectra. (d) Variations and fittings between Z' and the reciprocal square root of the angular frequency $(\omega)$ in the low frequency region of meso-PA/PmPD/GO, meso-PmPD/GO, and non$\mathrm{PA} / \mathrm{PmPD} / \mathrm{GO}$ in the mixture of furfuryl alcohol and the ternary electrolyte. 


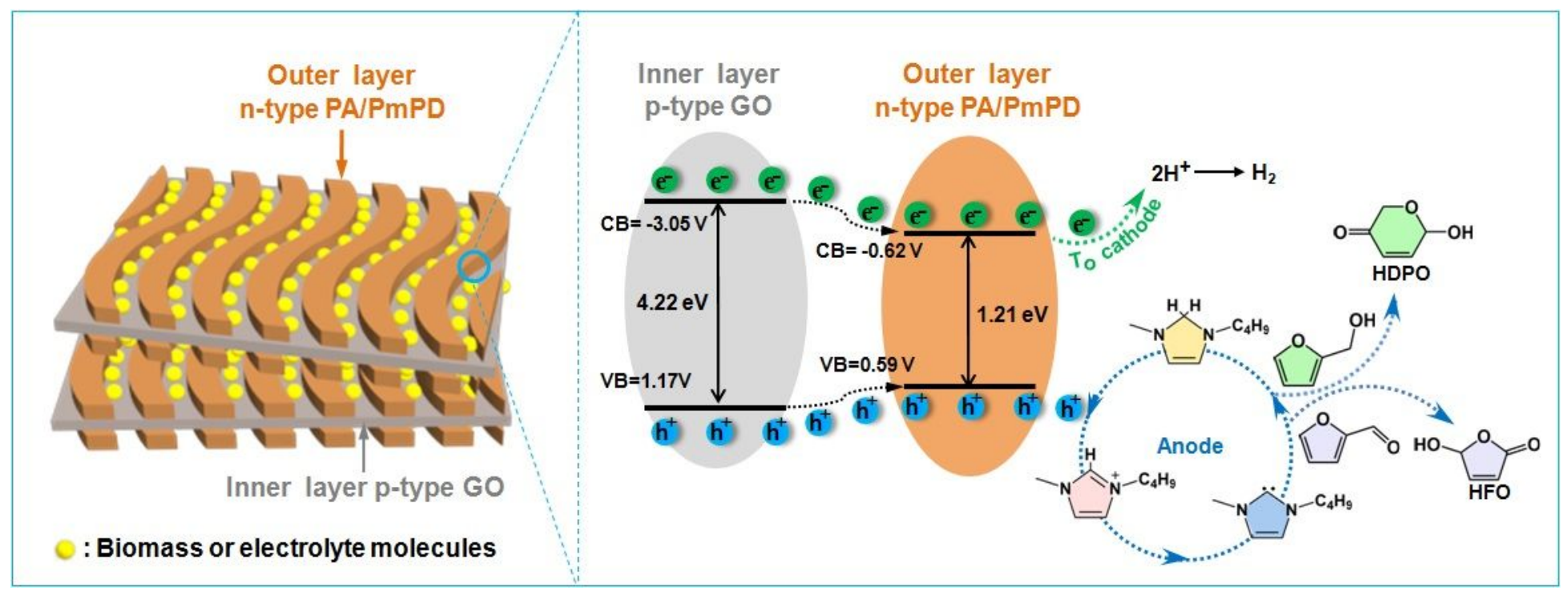

\section{Figure 8}

A possible mechanism of the charge separation and transfer at the $p$-n heterojunction interface of the meso-PA/PmPD/GO nanosheets during the electrocatalytic oxidation of furfuryl alcohol or furfural.

\section{Supplementary Files}

This is a list of supplementary files associated with this preprint. Click to download.

- Supplementarylnformation.pdf 